\title{
The cost-effectiveness of family/ family-based therapy for treatment of externalizing disorders, substance use disorders and delinquency: a systematic review
}

Maartje Goorden ${ }^{1 *+}$, Saskia J. Schawo ${ }^{1 \dagger}$, Clazien A.M. Bouwmans-Frijters ${ }^{1}$, Evelien van der Schee ${ }^{2}$, Vincent M. Hendriks ${ }^{2,3}$ and Leona Hakkaart-van Roijen'

\begin{abstract}
Background: Family therapy and family-based treatment has been commonly applied in children and adolescents in mental health care and has been proven to be effective. There is an increased interest in economic evaluations of these, often expensive, interventions. The aim of this systematic review is to summarize and evaluate the evidence on cost-effectiveness of family/family-based therapy for externalizing disorders, substance use disorders and delinquency.

Methods: A systematic literature search was performed in PubMed, Education Resource information Centre (ERIC), Psycinfo and Cochrane reviews including studies conducted after 1990 and before the first of August of 2013. Full economic evaluations investigating family/family-based interventions for adolescents between 10 and 20 years treated for substance use disorders, delinquency or externalizing disorders were included.

Results: Seven hundred thirty-one articles met the search criteria and 51 studies were initially selected. The final selection resulted in the inclusion of 11 studies. The quality of these studies was assessed. Within the identified studies, there was great variation in the specific type of family/family-based interventions and disorders. According to the outcomes of the checklists, the overall quality of the economic evaluations was low. Results varied by study. Due to the variations in setting, design and outcome it was not feasible to pool results using a meta-analysis.
\end{abstract}

Conclusions: The quality of the identified economic evaluations of family/family-based therapy for treatment of externalizing disorders, adolescent substance use disorders and delinquency was insufficient to determine the cost-effectiveness. Although commonly applied, family/family-based therapy is costly and more research of higher quality is needed.

Keywords: Systematic review, Cost-effectiveness, Family/family-based therapy, Delinquency, Substance use disorders

\footnotetext{
* Correspondence: Goorden@bmg.eur.nl

†'Equal contributors

${ }^{1}$ Institute for Medical Technology Assessment \& Institute of Health Policy \&

Management, Erasmus University Rotterdam, P.O. Box 17383000 DR

Rotterdam, The Netherlands

Full list of author information is available at the end of the article
} 


\section{Background}

Family therapy and family-based treatment is considered an evidence-based practice treatment for children and adolescents with externalizing disorders, symptoms of delinquency and/or substance use disorder [1,2]. Familial and extra-familial systems are known to influence the individual [3-7], and therefore family/family-based therapy is not only aimed at the individual youth but also at systems surrounding the individual. For instance, delinquency and substance abuse in adolescents have been shown to be influenced by family factors, like parenting style and attachment [3-7]. In addition, a recent review indicated that problems within the extra-familial system, like delinquent peers, problems with bonding at school and in the neighborhood are risk factors for delinquency and problem drinking [7]. As the individual, familial and extrafamilial systems are interconnected, family/familybased therapy not only positively affects the adolescent but also the family (family cohesion) and the extra-familial systems [8].

For the purpose of the present paper, family therapy and family-based treatment is broadly defined as treatments in which primarily family members and/or members of the families' wider networks are involved in the treatment process of resolving problems for young people [9] as opposed to treatments that mainly or solely focus on the individual youth, or treatments that do not focus on youths' problem behavior, like marital therapy.

Well-known forms of family/family-based treatments are Multisystemic therapy (MST) [10], Functional Family Therapy (FFT) [11] and Multidimensional Family therapy (MDFT) [12]. Although there is a large overlap between these types of therapies, there are also some differences [13]. For instance, in FFT and MST there is more focus on antisocial behavior. However, the degree of severity of the disorder is often higher in MST compared to FFT. More details of these differences are described in Appendix 1.

Recently, Von Sydow et al. [1] systematically reviewed studies on the effectiveness of family/family-based therapy for the treatment of children and adolescents who have externalizing disorders. Their study included disorders like substance abuse, attention deficit hyperactivity disorder, conduct disorder and symptoms of delinquency. They concluded that there is sound evidence that family/family-based therapy is effective with particularly large effect sizes for delinquency and substance abuse measures. However, in the meta analyses that were included in Von Sydow's systematic review, more cautious conclusions regarding the effectiveness of systemic therapy were drawn.

Current health care policy in the Netherlands and elsewhere places emphasis on the provision of effective mental health services in a cost effective way. Family/ family-based interventions are intensive as they consist of a relatively high number of sessions per week and subsequently are relatively expensive [14-16]. Therefore, there is a need for economic evaluations to assess whether additional effects gained through family/familybased therapy in comparison to alternative treatments if observed - justify the additional costs. Morgan et al. [17] described eight studies, analyzing the costeffectiveness of family-based treatments for substance abusing adults and adolescents and concluded that some of these treatments could be considered as cost-effective. However, family based therapies like marital therapy, were also included in this study. In addition, the literature search in this study was not systematically conducted and was only considering patients with substance use disorders. To our knowledge, no systematic review of economic evaluations of family/family-based therapy in externalizing, delinquent or substance-abusing adolescents has yet been performed.

Hence, this paper presents a systematic review of economic evaluations of systemic interventions in adolescents with externalizing disorders, substance abuse or delinquency. The aim of the present study was to assess the evidence on cost-effectiveness of family/family-based therapy for adolescents with externalizing disorders, substance use disorders or delinquency, and to evaluate the quality of the existing studies, and the generalizability of the study findings.

\section{Methods}

The review was performed according to the Cochrane handbook for systematic reviews of interventions [18] and adopted the Preferred Reporting for Systematic reviews and Meta-Analyses (PRISMA) statement [19].

\section{Search strategy}

A systematic literature search was performed in Pubmed, ERIC, Psycinfo and Cochrane reviews (including economic trials and clinical trials). These different search engines were used because of their high quality, coverage of large databases and their focus on economic trials.

Search terms encompassed the different types of systemic therapy (Functional Family Therapy, Multidimensional Family therapy, Multidimensional Foster Care, Multisystemic Therapy, Family Behavior Therapy and Brief Strategic Therapy) but also more general classifications (systemic therapy, substance abuse treatment, family based therapy, Family based intervention, Family system intervention, Family intervention program). These terms were searched for in title and abstract and were then combined with terms referring to economic evaluations searched for in title and abstract or a Medical Subject Headings $(\mathrm{MeSH})$ term (economic evaluation, costeffectiveness, cost-utility, cost benefit, cost analysis, cost 
measure) and in the title (costs). Costs were searched for only in the title, and not in the abstract, because the latter resulted in many irrelevant studies. This search term was included as we noticed that although in some studies both costs and effects were evaluated, the main focus of these studies was to evaluate the costs and a smaller part was referring to the effects. Consequently, when only terms referring to both the costs and effects were included, these studies would have been missed. The search term "Economic modeling" was not explicitly incorporated into the search strategy as the modeling should be part of a cost-effectiveness, cost utility, cost benefit or cost analysis (corresponding with our aim).

Abbreviations were also included. To improve our search, MeSH terms were used, see Appendix 2 for more details.

\section{Selection strategy}

In Fig. 1 the selection criteria are described and numbered. The criteria were applied to the studies in chronological order and when a study was excluded based on a criterion the number as shown in Fig. 1 was noted. We considered studies from January 1990 until January 2016. The selected study types were clinical/randomized controlled trials (RCT), reviews, systematic reviews and meta-analyses. The treatment needed to consist of a family/family-based intervention, targeted at adolescents (10-20 years old) with a substance use disorder, externalizing disorder or delinquent behavior. The method needed to be a cost or cost- effectiveness/benefit/ utility analysis. When studies were assessed for eligibility based on their abstracts and it was likely that they only contained cost-outcomes and no effect-outcomes, they were also included. To determine the eligibility of the full text articles, the same selection criteria were used, except that accessibility of the study was a requirement (full text available) and studies that only contained costs-outcomes and no effect-outcomes were excluded. The selection of the articles was performed by two researchers independently. Differences in selections were discussed until consensus was reached.

\section{Data extraction and risk of bias}

The quality of the studies was assessed with the British Medical Journal Checklist for authors and peer reviewers of economic submissions [20] and the Consensus on Health Economic Criteria (CHEC) list for assessment of methodological quality of economic evaluations [21] as recommended by the Cochrane reviews handbook [18]. We also consulted the critical appraisal of the studies by the NHS Economic Evaluation Database (NHS EED) structured abstract [18]. This is a database from Cochrane library consisting of structured abstracts of economic evaluations of health care interventions. Full economic evaluations were identified from a variety of sources and assessed according to a set of quality criteria. Subsequently, detailed structured abstracts were produced. In addition to the checklists, information about the economic perspective of the study (health care, societal etc.), design, country, follow-up, type of disorder, sample size, study dropout, age, gender, type, duration and intensity of intervention, time horizon, currency and price year, key features of sensitivity analyses and the included cost types were collected for the economic evaluation described in the studies. In accordance with the suggestions in the Cochrane handbook [18] five different biases of the individual studies were addressed: selection bias, performance bias, detection bias, attrition bias and reporting bias [18]. They were respectively addressed by assessing if patients were properly balanced at baseline, patients and therapists were blinded, outcome assessors were blinded, the amount of dropout in the studies and by reading the protocols of the studies.

\section{Results}

A total of 731 articles met the search criteria. After removal of duplicates and a first selection based on the abstracts, 51 studies matched the inclusion criteria. After assessment for eligibility, 11 studies were selected (see Fig. 1).

\section{Characteristics of the studies}

An overview of the characteristics of the studies, participants and the interventions is shown in Table 1. Ten of the eleven selected studies were published between 2003 and 2015 [22-31] and one study was published in 1996 [32]. Eight of the studies originated from the United States (USA) [22-24, 27, 29-32]. Remaining studies were initiated in Sweden [26], England [28] and Mexico [25]. All studies were (based upon) randomized controlled trials. Two pairs of studies [22, 24, 27, 29] were each based on one sample. Most of the studies compared a family/family-based intervention with care as usual [23, 26, 28, 30-32]. MST was the most researched intervention as it was investigated in eight studies [23, 24, 26-28, 30-32]. In the Study of Borduin et al. [31] Multisystemic Therapy for Problem Sexual Behavior (MST-PSB) was investigated. MST-PSB is an adaptation to MST aimed at the treatment of juvenile sexual offenders. A description of the (non- family/family-based) comparator interventions is shown in Table 2. The mean number of sessions of the family/family-based interventions was between 1 and 3 times a week and the mean duration of treatment was between 12 and 31 weeks. The average follow-up time was between 6 and 300 months ( 25 years); only four studies followed patients for more than 1 year $[26,28,30,31]$. Two studies were outliers in respect to the time horizon they used (8 years and 25 years) [30, 31]. 

Selection/eligibility criteria
1. Year of publication
2. Publication:
After 1990
3. a Treatment:
Systematic Review, randomized/clinical trial, meta-analysis
b Population:
family/family-based
c Outcome:
Adolescents, $10-20$ years of age
Cost-effectiveness/benefit/utility, costs
4. Disease/symptoms:
delinquency, substance use disorders, externalizing Disorders

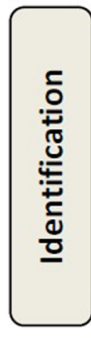

Records identified through database searching

$(n=731)$

Pubmed: $n=345$

Psycinfo: $n=276$

Eric: $n=28$

Cochrane: $n=82$

Additional records identified

through other sources

$(n=0)$

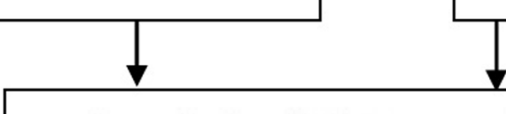

Records after duplicates removed

$(n=602)$
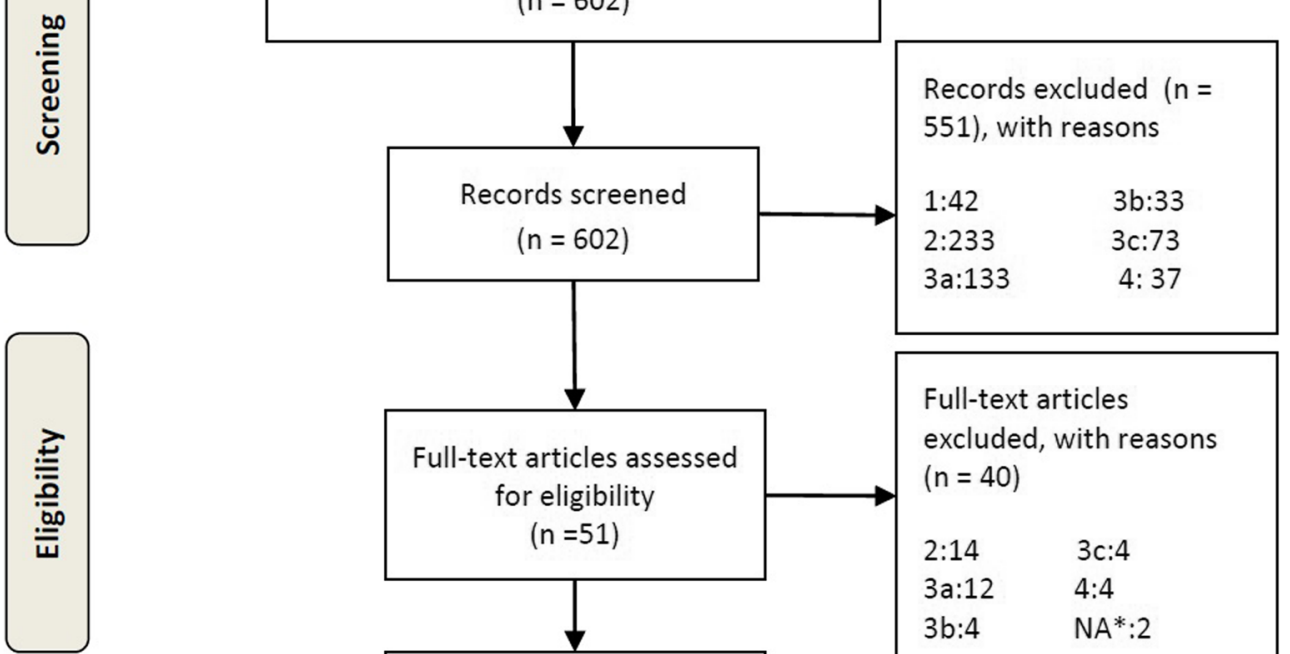

Full-text articles excluded, with reasons ( $n=40$ )

Full-text articles assessed for eligibility ( $n=51$ )

$2: 14 \quad 3 c: 4$

3a:12 $4: 4$

$3 b: 4 \quad N A *: 2$

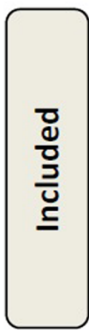

Studies included in

qualitative synthesis

$(n=11)$

뭉

Studies included in quantitative synthesis $(n=11)$

*NA=Not available; Two studies were not available

Fig. 1 PRISMA flow diagram [19] 
Table 1 Features of the studies, participants and the interventions

\begin{tabular}{|c|c|c|c|c|c|c|c|c|c|c|c|c|c|c|c|c|}
\hline \multirow[t]{3}{*}{ Study } & \multicolumn{3}{|c|}{ Features study } & \multicolumn{9}{|c|}{ Features participants } & \multicolumn{4}{|c|}{ Features intervention } \\
\hline & \multirow[t]{2}{*}{ Coun-try } & \multirow[t]{2}{*}{$\begin{array}{l}\text { Follow-up } \\
\text { (months) }\end{array}$} & \multirow[t]{2}{*}{ Design } & \multirow[t]{2}{*}{ Disorder } & \multicolumn{2}{|c|}{$\begin{array}{l}\text { Sample } \\
\text { size }\end{array}$} & \multicolumn{2}{|c|}{$\begin{array}{l}\text { Completed } \\
\text { study }\end{array}$} & \multicolumn{2}{|c|}{ Age } & \multicolumn{2}{|c|}{$\begin{array}{l}\text { Sex } \\
\text { (\% male) }\end{array}$} & \multicolumn{2}{|l|}{ Intervention } & \multirow{2}{*}{$\begin{array}{l}\text { Number } \\
\text { of sessions } \\
\text { per week }\end{array}$} & \multirow{2}{*}{$\begin{array}{l}\text { Treatment } \\
\text { duration } \\
\text { (weeks) }\end{array}$} \\
\hline & & & & & I & 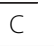 & I & C & I & $\bar{C}$ & I & $\mathrm{C}$ & I & $\mathrm{C}$ & & \\
\hline $\begin{array}{l}\text { Schoenwald et al., } \\
1996 \text { [32] }\end{array}$ & USA & 6 AT & $\mathrm{RCT}$ & SUD & 59 & 59 & NS & & 16 & & 79 & & MST & CAU & $2-3^{b}$ & $18-19$ \\
\hline \multirow[t]{8}{*}{ French et al., 2003 [22] } & USA & $12^{\mathrm{a}}$ & RCT & SUD & & & & & & & & & Trial 1: & & & \\
\hline & & & & & 102 & & 564 & & 16 & & 81 & & MET/CBT5 & & $0-1$ & $6-7$ \\
\hline & & & & & 96 & & & & 16 & & 86 & & MET/CBT12 & & $0-1$ & $12-14$ \\
\hline & & & & & 102 & & & & 16 & & 84 & & FSN & & $1-2$ & $12-14$ \\
\hline & & & & & & & & & & & & & Trial 2: & & & \\
\hline & & & & & 100 & & & & 16 & & 79 & & MET/CBT5 & & $0-1$ & $6-7$ \\
\hline & & & & & 100 & & & & 16 & & 80 & & ACRA & & $1-2$ & $12-14$ \\
\hline & & & & & 100 & & & & 16 & & 85 & & MDFT & & $1-2$ & $12-14$ \\
\hline Sheidow et al., 2004 [23] & USA & 12 AT & $\mathrm{RCT}$ & PC & 115 & & NS & & 13 & & 67 & & MST & CAU & NS & 16 \\
\hline \multirow[t]{11}{*}{ Dennis et al., 2004 [29] } & USA & 12 & $\mathrm{RCT}$ & SUD & & & 564 & & & & & & Trial 1: & & & \\
\hline & & & & & 102 & & & & 16 & & & & $\mathrm{MET} / \mathrm{CBT}$ & & $0-1$ & $6-7$ \\
\hline & & & & & 96 & & & & 16 & & & & 5 & & $0-1$ & $12-14$ \\
\hline & & & & & 102 & & & & 16 & & & & $\mathrm{MET} / \mathrm{CBT}$ & & $1-2$ & $12-14$ \\
\hline & & & & & & & & & & & & & 12 & & & \\
\hline & & & & & 100 & & & & 16 & & & & FSN & & $0-1$ & $6-7$ \\
\hline & & & & & 100 & & & & 16 & & & & Trial 2: & & $1-2$ & $12-14$ \\
\hline & & & & & 100 & & & & 16 & & & & $\mathrm{MET} / \mathrm{CBT}$ & & $1-2$ & $12-14$ \\
\hline & & & & & & & & & & & & & 5 & & & \\
\hline & & & & & & & & & & & & & ACRA & & & \\
\hline & & & & & & & & & & & & & MDFT & & & \\
\hline \multirow{4}{*}{$\begin{array}{l}\text { McCollister et al., } \\
2009 \text { [24] }\end{array}$} & USA & 12 & RCT & SUD & 38 & 42 & NS & & 1 & 1 & 84 & 81 & DC & FC & $N S^{1}$ & $\mathrm{NS}^{1}$ \\
\hline & & & & & 38 & & & & 5 & 5 & 84 & & $\mathrm{DC}+\mathrm{MST}$ & & & \\
\hline & & & & & 43 & & & & 15 & & 4 & & $\mathrm{DC}+\mathrm{MST}+\mathrm{CM}$ & & & \\
\hline & & & & & & & & & 15 & & & & & & & \\
\hline \multirow[t]{3}{*}{ French et al., 2008 [25] } & MEX & 7 & RCT & SUD & 30 & 30 & 114 & & 16 & 1 & 80 & 83 & FFT & group & $N S^{1}$ & $N S^{1}$ \\
\hline & & & & & 29 & & & & 16 & 6 & 76 & & Joint & & & \\
\hline & & & & & 31 & & & & 16 & & 84 & & CBT & & & \\
\hline Olsson, 2010 [26] & SW & 24 & RCT & $C D$ & 79 & 77 & NS & & 15 & & 61 & & MST & CAU & NS & $12-20$ \\
\hline \multirow[t]{3}{*}{ Sheidow et al., 2012 [27] } & USA & 12 & RCT & SUD & 38 & 4 & 29 & 33 & 15 & & 83 & & DC & FC & $\mathrm{NS}^{1}$ & $\mathrm{NS}^{1}$ \\
\hline & & & & & 38 & 2 & 29 & & & & & & $\mathrm{DC}+\mathrm{MST}$ & & & \\
\hline & & & & & 43 & & 37 & & & & & & $\mathrm{DC}+\mathrm{MST}+\mathrm{CM}$ & & & \\
\hline \multirow[t]{2}{*}{ Cary et al., 2013 [28] } & ENG & 30 & RCT & DEL & 56 & 52 & 46 & 45 & 15 & 15 & 83 & 82 & MST+ & CAU & 3 & 20 \\
\hline & & & & & & & & & & & & & CAU & & & \\
\hline Dopp et al. (2014) [30] & USA & 300 & RCT & DEL & 92 & 84 & 70 & 56 & 15 & & 69 & & MST & CAU & $3-4$ & 21 \\
\hline Borduin et al. (2015) [31] & USA & 107 & RCT & DEL & 24 & 24 & 24 & 22 & 14 & & 96 & & MST-PSB & CAU & 3 & 31 \\
\hline
\end{tabular}

Legend: I intervention, C comparator, NS not stated, $N S^{1}$ reference to non-accessible article, NA not applicable, USA United States of America, SW Sweden, ENG England, MEX Mexico, SUD substance use disorder, CD conduct disorder, $P C$ psychiatric crisis, MST multisystemic therapy, Joint combination of individual and family therapy, group skill-focused psycho-education group intervention, IT individual treatment, MST-PSB MST for Problem Sexual Behavior, CAU care as usual, FSN family support network, MDFT multidimensional family treatment, MET/CBT12 motivational enhancement treatment/cognitive behavior therapy, 12 sessions; $M E T /$ CBT5 motivational enhancement treatment/cognitive behavior therapy, 5 sessions; $A C R A$ adolescent community reinforcement approach, $D C$ drug court with community services, $D C+M S T$ drug court with multisystemic therapy, $D C+M S T+C M$ drug court with mst and enhanced with a contingency management programs, FFT functional family therapy, FC family court with community services

${ }^{a}$ Cost data was only collected only during 3-9 months

${ }^{\mathrm{b}}$ The intensity of the treatment was between 2 and 3 times a week; $A T$ after treatment 
Table 2 Descriptions of comparator interventions

\begin{tabular}{ll}
\hline FSN & Cognitive behavioral sessions and motivation treatment \\
in combination with a family component. \\
MET/CBT5 & $\begin{array}{l}\text { Motivational component and a cognitive behavioral } \\
\text { component, to enhance motivation to change drug } \\
\text { abuse and to grow the skills to maintain and regulate } \\
\text { abstinence }\end{array}$ \\
MET/CBT12 & MET/CBT5+ 7 sessions of CBT are added to the therapy. \\
FC & Family court treatment with community services/ \\
& Appearance court 2 times a year/ outpatient alcohol \\
& and drug abuse service from the local center of the \\
& state's substance abuse commission \\
& Drug court treatment with community services/ \\
& Appearance court 1 time a week/ outpatient alcohol \\
and drug abuse service from the local center of the \\
state's substance abuse commission and monitoring \\
drug abuse \\
Frequent in home screens for drug use, voucher \\
system contingent on clean screens, and drug \\
refusal training. \\
Identifying reinforces that are incompatible with \\
the drug use and to strengthen those \\
Sheidow et al. [23]: admission to a psychiatric unit \\
and aftercare \\
Schoenwald et al. [32]: outpatient substance abuse \\
Services \\
Olsson et al. [26]: Not described \\
Cary at al. [28]: Youth Offending Team (YOT) \\
Dopp et al. [30]; Individual Therapy (IT) \\
Borduin et al. [31]: Cognitive behavioral group therapy \\
and individual services (from local juvenile court)
\end{tabular}

FSN family support network, MET/CBT5 motivational enhancement treatment/ cognitive behavior therapy, 5 sessions, MET/CBT12 motivational enhancement treatment/cognitive behavior therapy, 12 sessions; ACRA adolescent community reinforcement approach, $F C$ family court with community services, $D C$ drug court with community services, $C M$ contingency management programs, CAU care as usual

Six studies were aimed at adolescents with substance use disorder [22, 24, 25, 27, 29, 32], one study investigated adolescents with a conduct disorder [26], one study adolescents at risk for continuing criminal activity [28],one study adolescents who had experienced a psychiatric crisis [23], another study adolescents who were serious juvenile offenders [30] and one study aimed to investigate juvenile sexual offenders [31]. The average sample size of the 9 studies (with separate samples) was $178(\mathrm{SD}=163)$ with a variation between 48 and 600 patients. Follow-up attrition, when registered, was low (not more than $30 \%$ ). Average age at baseline was 15 (Standard Deviation $(\mathrm{SD})=1$ ) years and between 61 and $96 \%$ of the individuals were males.

Types of economic analyses included cost-effectiveness analyses [23, 25, 27, 29], cost-benefit analyses [22, 26, 30, 31] and cost offset analyses [28]. The difference between a costoffset and a cost-benefit analysis is often not well-explained. A cost-offset analysis compares the monetary value of resource use with the monetary value of costs reduced by the intervention (usually health care costs). In contrast to a cost-benefit analysis which also focusses on other outcomes that are translated in monetary outcomes (like translating number of life years gained to a monetary value). In reality, cost-offset analysis is a partial cost-benefit analysis because it compares the cost of a program with the monetary value of a single outcome (i.e., avoided future health care costs). In two studies, the economic evaluation was not explicitly classified [24, 32].

\section{Outcomes of the studies}

Details of the interventions and outcomes of our analyses are described in Tables 3 and 4. Costs were indexed until 2014.

\section{Substance abuse}

Six studies were identified which included adolescents that were treated for substance abuse [22, 24, 25, 27, 29, 32]. Three of these studies considered costs and effects $[25,27,29]$ and three considered both costs and benefits [22, 24, 32].

Studies considering costs and effects In the study of French et al. [25] FFT was shown to be more costeffective than a skill-focused psycho-education group intervention for treating substance use disorders and delinquency after the first 4 months. After 12 months no such effect was observed. Therefore, after 12 months the cost-effectiveness analysis reduced to a simple cost minimization analysis. As only treatment costs were considered (narrow perspective), the intervention with the lowest intervention costs, in this case group therapy, was considered to be economically beneficial. In another study, Dennis et al. [29] computed cost-effectiveness ratios and these ratios indicated that overall, the most cost-effective interventions were Motivational Enhancement Treatment/ Cognitive Behavior Therapy, 5 sessions (MET/CBT5) and Motivational enhancement treatment/ Cognitive Behavior Therapy, 12 sessions (MET/CBT12) when compared to Family Support Network (FSN) in Trial 1 and Adolescent Community Reinforcement Approach (ACRA) and MET/CBT5 when compared to MDFT in Trial 2. Sheidow et al. [27], computed Average Cost-Effectiveness Ratios (ACERS). ACERS only incorporate the pre-post treatment effect of one single treatment so treatments are not directly compared. Although this study showed that Drug Court with community services (DC) was more cost effective compared to FC regarding substance use disorders and that the addition of multi-systemic therapy (MST) resulted in an economically more beneficial treatment, the treatments were not directly compared [27]. 
Table 3 Studies that reported substance use disorders

Studies considering costs and effects of substance abuse

\begin{tabular}{|c|c|c|c|c|c|}
\hline \multirow{6}{*}{$\begin{array}{l}\text { Dennis } \\
\text { (2004) [29] }\end{array}$} & & \multirow{2}{*}{\multicolumn{2}{|c|}{$\begin{array}{l}\text { Costs intervention and comparators (per episode of care per patient) (MET/CBT 5, MET/CBT 12, FSN, ACRA, MDFT) } \\
\text { In trial } 1 \mathrm{MET} / \mathrm{CBT} \text { 5,MET/CBT } 12 \text { and FSN were compared. In trial } 2 \mathrm{MET} / \mathrm{CBT} 5, \text { ACRA and MDFT were } \\
\text { compared. Costs were collected with a program (DATCAP) which yields estimates such as the total annual } \\
\text { opportunity cost of treatment and the labor cost per client. }\end{array}$}} & \multirow{3}{*}{\multicolumn{2}{|c|}{$\begin{array}{l}\text { Difference cost } \\
\text { The difference in costs were not showed } \\
\text { in this study. } \\
\text { However, it was showed that the } \\
\text { differences were significant. }\end{array}$}} \\
\hline & & & & & \\
\hline & & $\begin{array}{l}\text { MET/CBT } 5 \text { (trial 1): } \\
€ 1,226 \\
\text { MET/CBT } 12 \text { (trial 1): } \\
€ 1,305 \\
\text { FSN (trial 1): } \\
€ 3,576\end{array}$ & $\begin{array}{l}\text { MET/CBT } 5 \text { (trial 2): } \\
€ 1,716 \\
\text { ACRA (trial 2): } \\
€ 1,551 \\
\text { MDFT (trial 2): } \\
€ 2,205\end{array}$ & & \\
\hline & & \multicolumn{2}{|c|}{ Effects intervention and comparators (per patient) (MET/CBT 5, MET/CBT 12, FSN, ACRA, MDFT) } & \multicolumn{2}{|l|}{ Difference effects } \\
\hline & & \multicolumn{2}{|l|}{$\begin{array}{l}\text { Met CBT } 5 \text { (trial 1) } \\
\text { Days of abstinence: } 269 \\
\text { Recovery*: } 28 \% \\
\text { Met CBT } 12 \text { (trial 1) } \\
\text { Days of abstinence: } 256 \\
\text { Recovery: } 17 \% \\
\text { MET FSN (trial 1) } \\
\text { Days of abstinence: } 260 \\
\text { Recovery: } 22 \% \\
\text { *Recovery is defined as having no use or abuse } \\
\text { dependence problems and living in the community }\end{array}$} & \multicolumn{2}{|c|}{$\begin{array}{l}\text { The difference in effects were not showed } \\
\text { in this study } \\
\text { However it was showed that the difference } \\
\text { was not significant. }\end{array}$} \\
\hline & Results & $\begin{array}{l}\text { Cost per day of abstinence: } \\
\text { Met CBT5 (trial 1): } € 541 \\
\text { Met CBT 12: } € 677 \\
\text { Met FSN: } € 1,667 \\
\text { Costs per person in recovery } \\
\text { Met CBT5 (trial 1): } € 4,360 \\
\text { Met CBT 12: } € 41,172 \\
\text { Met FSN: } € 16,651\end{array}$ & & \multicolumn{2}{|l|}{$\begin{array}{l}\text { Cost per days of abstinence: } \\
\text { MET/CBT5 (trial 2): } € 991 \\
\text { ACRA: } € 729 \\
\text { MDFT: } € 1,143 \\
\text { Costs per person in recovery } \\
\text { MET/CBT5 (trial } 2): € 7,337 \\
\text { ACRA: } € 4,913 \\
\text { MDFT: } € 12,970\end{array}$} \\
\hline \multirow{4}{*}{$\begin{array}{l}\text { French } \\
\text { (2008) [25] }\end{array}$} & & \multirow{2}{*}{$\begin{array}{l}\text { Costs intervention per patient (FFT, Joint and CBT) } \\
\text { FFT: } \\
\text { Treatment costs: } € 1,817 \\
\text { Joint: } \\
\text { treatment costs: } € 2,847 \\
\text { CBT: } \\
\text { Treatment costs: } € 1.439\end{array}$} & \multirow{2}{*}{$\begin{array}{l}\text { Costs comparator per } \\
\text { patient (Group) } \\
\text { Group: } \\
\text { Treatment costs: } € 990\end{array}$} & \multicolumn{2}{|l|}{ Difference costs } \\
\hline & & & & \multicolumn{2}{|c|}{$\begin{array}{l}\text { The difference in costs were not showed } \\
\text { in this study }\end{array}$} \\
\hline & & \multirow[b]{2}{*}{$\begin{array}{l}\text { Effects intervention per patient (FFT, Joint and CBT) } \\
\text { FFT: } \\
\text { \% days marijuana use } 4 \text { months: } 25.3 \\
\text { \%of days marijuana use } 7 \text { months:39.8 } \\
\text { YSR delinquency score } 4 \text { months: } 8.2 \\
\text { YSR delinquency score } 7 \text { months:9.2 } \\
\text { Joint } \\
\% \text { of days of marijuana use } 4 \text { months: } 38.1 \\
\text { marijuana use } 7 \text { months:35.4 } \\
\text { YSR delinquency score } 4 \text { months: } 9.1\end{array}$} & \multirow[b]{2}{*}{$\begin{array}{l}\text { Effects comparator per } \\
\text { patient (Group) } \\
\text { Group } \\
\% \text { of days of marijuana use } \\
4 \text { months: } 54.8 \\
\text { marijuana use } 7 \text { months: } 40.7 \\
\text { YSR delinquency score } \\
4 \text { months: } 9.5 \\
\text { IYSR delinuency score } \\
7 \text { months: } 9.4\end{array}$} & \multicolumn{2}{|c|}{ Difference effects with regression model: } \\
\hline & & & & $\begin{array}{l}\text { FFT versus group: } \\
\% \text { days marijuana use after } \\
4 \text { months: }-20.11^{*} \\
\% \text { days marijuana use after } \\
7 \text { months: } 4.87 \\
\text { YSR delinquency score } \\
4 \text { months: }-0.60 \\
\text { YSR delinquency score } \\
7 \text { months: } 0.15\end{array}$ & $\begin{array}{l}\text { Joint versus } \\
\text { group } \\
\text { Delinquency } \\
\text { score after } 4 \\
\text { months: }-0.50 \\
\text { Delinquency } \\
\text { score after } 7 \\
\text { months: }-1.50\end{array}$ \\
\hline
\end{tabular}

\begin{abstract}
Met CBT 5 (trial 1)
Met CBT 12 (trial 1)

Days of abstinence: 260

Recovery: $22 \%$

Cost per day of abstinence:

Met CBT5 (trial 1): $€ 541$

Met FSN: $€ 1,667$
\end{abstract}

Recovery is defined as having no use or abus
FFT:
Treatment costs: $€ 1.43$
ects intervention per patient (FFT, Joint and CB
\% of days of marijuana use 4 months: 38.1
YSR delinquency score 4 months: 9.

ent (Group)

$\%$ of days of marijuana use

4 months: 54.8

IYSR delinuency score 
Table 3 Studies that reported substance use disorders (Continued)

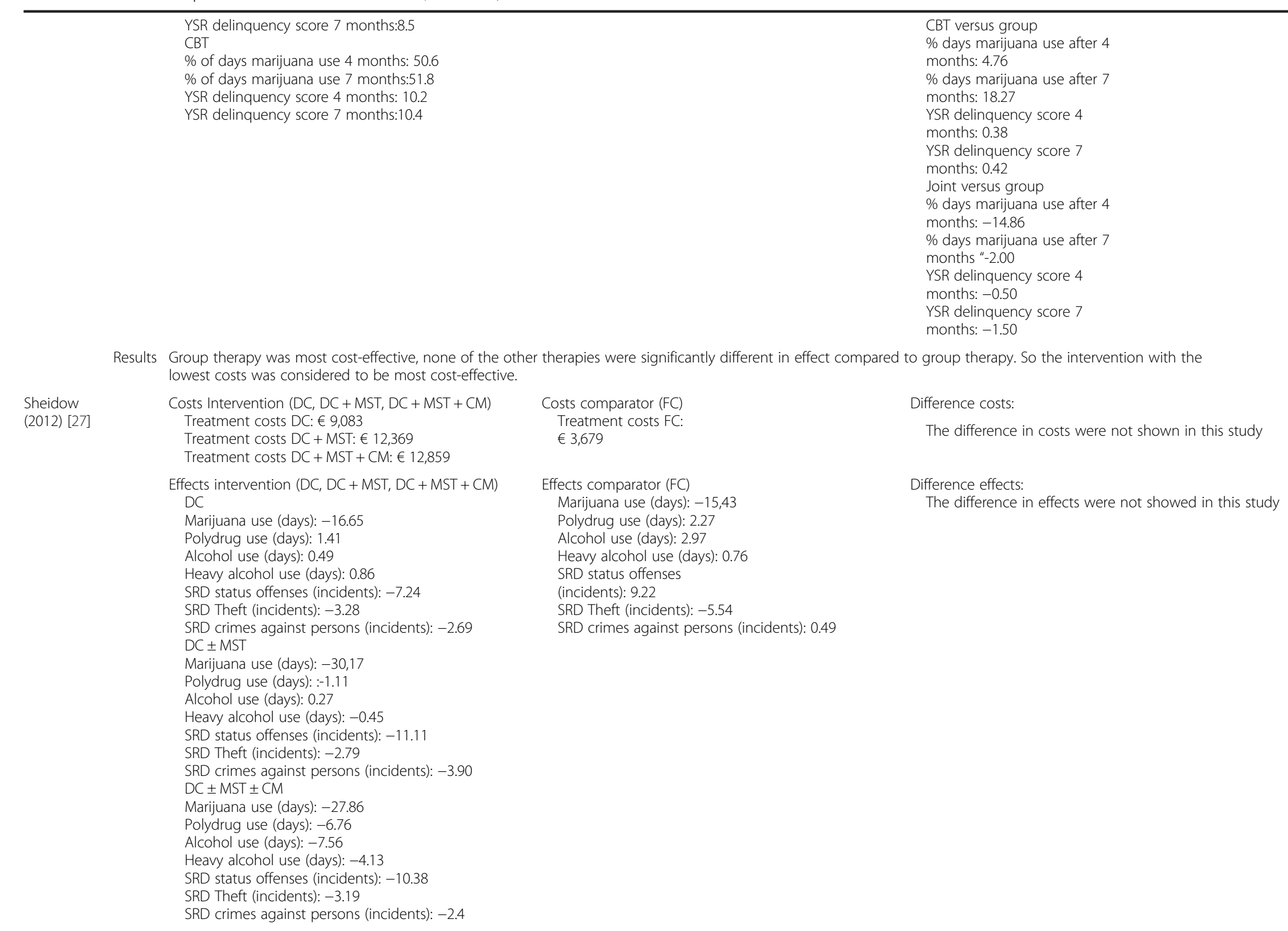

therapies were significantly different in effect compared to group therapy. So the intervention with the

Costs Intervention (DC, DC + MST, DC + MST + CM)

costs DC: $€ 9,083$

Costs comparator ( $\mathrm{FC})$

Treatment costs FC:

fects comparator (FC)

Marijuana use (days): $-15,43$

RD Theft (incidents): -5.54

RRD status offenses (incidents): -7.24

SRD crimes against persons (incidents): -2.69

eavy alcohol use (days): -0.45

RD status offenses (incidents): -11.11

$\mathrm{DC} \pm \mathrm{MST} \pm \mathrm{CM}$

SRD status offenses (incidents): -10.38

SRD crimes against persons (incidents): -2.4 
Table 3 Studies that reported substance use disorders (Continued)

Results ACERS were calculated; average costs/ difference between mean incidents before and after treatment (negative means inefficient)

Marijuana use:
Polydrug use:
Alcohol use:
Heavy alcohol use:
SRD status offenses:
SRD theft:
SRD crimes against persons:

SRD crimes against persons:

$\begin{array}{ll}F C & D C \\ € 238 & € 545 \\ (215-262) & (474-617) \\ €-1,619 & €-6,425 \\ (-8,8839-5,601) & (-27,541-14,692) \\ €-, 1,239 & €-18,814 \\ (-6,546-5,601) & (-42,034-4,405) \\ €-4,857 & €-10,535 \\ (-10,632-918) & (-28,804-7,733) \\ €-400 & € 1,254 \\ (-1,206-398) & (1,132-1,376) \\ € 663 & € 2,773 \\ (428-899) & (-2.441-7,987) \\ €-7,588 & € 3,377 \\ (-10,667-4,510) & (2,976-3,777)\end{array}$

Studies considering costs and benefits of substance abuse

Schoenwald Costs interventions (MST)

(1996) [32]

Mental health outpatient (total): $€ 4,242$

Mental health day treatment (total): $€ 5,423$

Mental health residential treatment (total): $€ 6,899$

Psychiatric inpatient (total): $€ 15,752$

Psychiatric emergency room (total): $€ 1,150$

Substance abuse outpatient (total): $€ 2,001$

Substance abuse residential treatment (total): $€ 3,450$

Substance abuse inpatient (total): $€ 16,098$

Marine Institute day treatment (total): $€ 18,926$

Marine Institute residential treatment (total): $€ 3,036$

Treatment costs: $€ 266,516$

DC + MST
$€ 410$
$(377-442)$
$€ 11,209$
$(-3,757-26,175)$
$€-44,838$
$(-61,014-28,662)$
$€ 27,592$
$(-14,636-69,821)$
$€ 1,114$
$(907-1,321)$
$€ 4,428$
$(-1,224-10,081)$
$€ 3,175$
$(236-6,123)$

Costs comparator (CAU)

Mental health outpatient

(total): $€ 19,075$

Mental health day treatment

(total): $€ 1,118$

Mental health residential treatment (total): $€ 0$

Psychiatric inpatient (total): $€ 18,513$

Psychiatric emergency room

(total): $€ 3,450$

Substance abuse outpatient

(total): €20,272

Substance abuse residential treatment (total): $€ 43,695$

Substance abuse inpatient

(total): €93,771

Marine Institute day treatment (total): $€ 28,618$

Marine Institute residential treatment (total):
$\mathrm{DC}+\mathrm{MST}+\mathrm{CM}$

$€ 461$

$(434-488)$

$€ 1,912$

$(1,624-2,182)$

$€ 1,699$

$(1,486-1,912)$

$€ 3,109$

$(1,708-4,511)$

$€ 1,239$

$(1,009-1,496)$

$€ 4,032$

$(1,204-6,859)$

$€ 5,346$

$(4,723-5,968)$

Benefits intervention

Incarceration days:

$€ 120,851$

Results MST: Total costs (costs + benefit) with incarceration $=€ 408,919$ and the total costs (costs + benefit) with incarceration per youth $=€ 6,930$

CAU: Total costs (costs + benefit) with incarceration $=€ 335,845$ and the costs (costs + benefit) per youth $=€ 5,693$.

Difference in total between groups $=€ 1,019$

French

Costs interventions (MET/CBT 5, MET/CBT 12, FSN, ACRA, MDFT)

Benefits interventions (MET/CBT 5, MET/CBT 12, FSN, ACRA, MDFT)

Treatment costs were measured

Health service utilization; Outpatient clinic/doctor's office visit

Days bothered by health/medical problem

Substance-absue treatment utilization; Days in

detoxification program; Day in inpatient

treatment program; Day in long-term

residential program; Intensive outpatient program visits:

Regular outpatient program visits

Education and employment; Days missed at school

or training; Personal income; Days stressful for parents 
Table 3 Studies that reported substance use disorders (Continued)

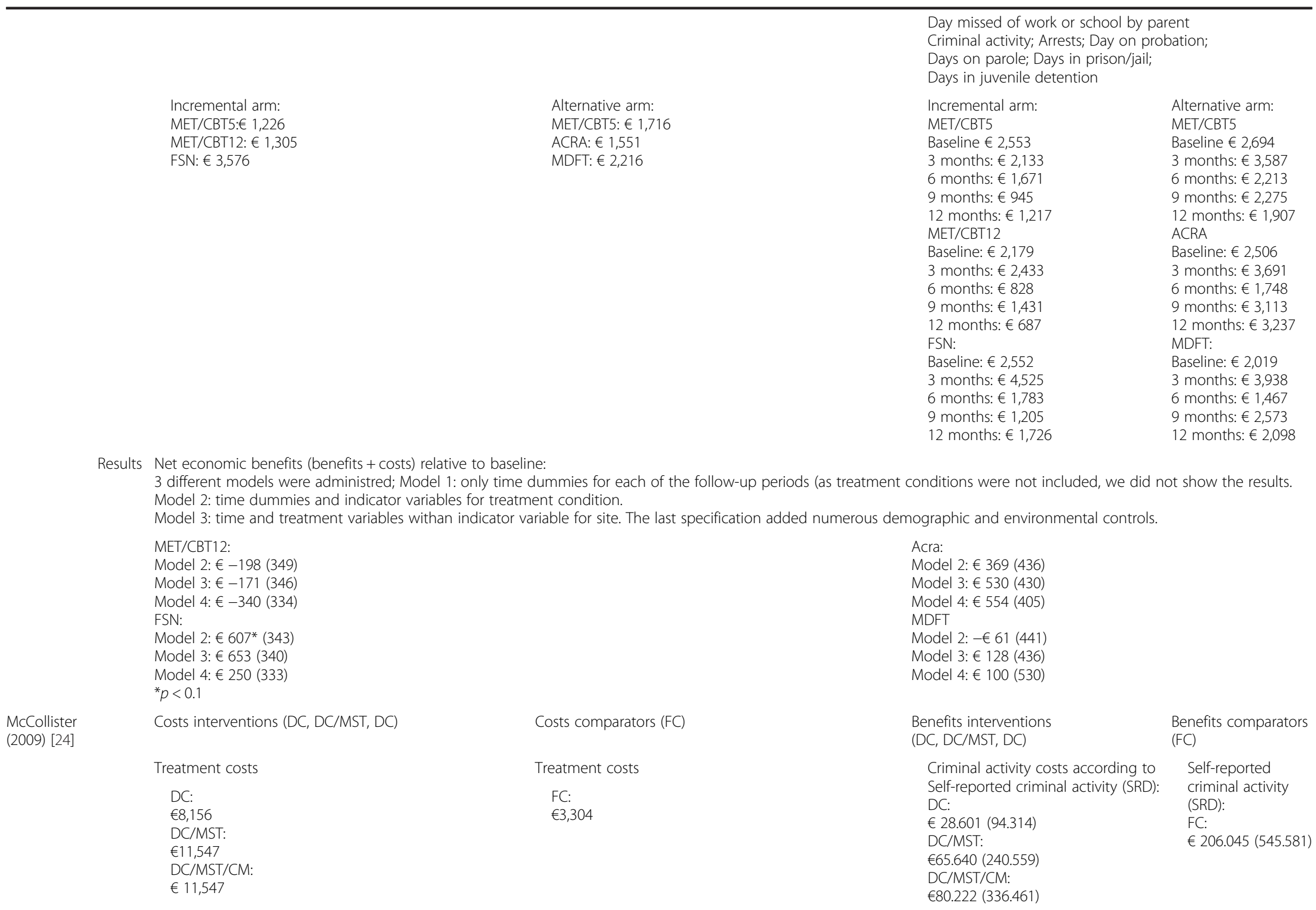


Table 3 Studies that reported substance use disorders (Continued)

Results After 12 months, total costs relative to FC with multivariate model (intervention costs not incorporated)

DC: $€-124,877(-84,107)$

DC/MST: $€-117,918(-82,570)$

DC/MST/CM: $€ 140,274(/ 79.066)^{*}$

All DC conditions generated reduction in crime costs, greater than average costs of treatment.

Currency and price year: Sheidow (2004).USD, 1997; Dennis (2004).USD, 1999; French 2008.USD, 1998; Sheidow (2012).USD 2004. When a price year was not stated it was estimated by taking the mean year of the

study duration or when not available subtracting 1 from the year of publication of the study

MST multisystemic therapy, Joint combination of individual and family therapy, group skill-focused psycho-education group intervention, CAU care as usual, FSN family support network, MDFT multidimensional family treatment, $M E T / C B T / 2$ motivational enhancement treatment/cognitive behavior therapy, 12 sessions, MET/CBT5 motivational enhancement treatment/cognitive behavior therapy, 5 sessions, Acra adolescent community reinforcement approach, DC drug court with community services, DC+MST drug court with multisystemic functional family therapy, FC family court with community services, ACERS average cost-effectiveness ratios 
Table 4 Studies considering externalizing disorders and delinquency Studies considering both costs and effects

\begin{tabular}{|c|c|c|c|c|}
\hline \multirow{5}{*}{$\begin{array}{l}\text { Sheidow } \\
\text { (2004) [23] }\end{array}$} & \multirow{3}{*}{$\begin{array}{l}\text { Costs intervention (MST) } \\
\text { Medicaid (government insurance program) } \\
\text { costs (inpatient, Outpatient, Pharmacy, } \\
\text { other costs), } \\
\text { Other treatment costs paid for by study } \\
\text { MST } \\
\text { Medicaid costs: } 0 \text {-end treatment } \\
\text { ( } 4 \text { months): } € 9,311 \\
( \pm 7,755) \\
\text { Medicaid costs: End treatment- } 12 \text { months: } \\
€ 13,237 \text { ( } \pm 15,144) \\
\text { Other treatment costs paid for by } \\
\text { study: } € 11,617\end{array}$} & \multirow{3}{*}{$\begin{array}{l}\text { Costs comparator (CAU) } \\
\text { Medicaid (government insurance program) } \\
\text { costs (inpatient, Outpatient, Pharmacy, } \\
\text { other costs), Other treatment costs } \\
\text { paid for by study } \\
\text { CAU } \\
\text { Medicaid costs: } 0 \text {-end treatment ( } 4 \text { months): } \\
€ 13,255 \text { ( } \pm 5,762 \text { ) } \\
\text { Medicaid costs: End treatment-12 months: } \\
€ 15,207 \text { ( } \pm 18,485) \\
\text { Other treatment costs paid for by study: } € 0\end{array}$} & \multicolumn{2}{|c|}{ Difference costs (Costs CUU -Costs ${ }_{M S T}$ ) (after risk adjusted model): } \\
\hline & & & & $-€ 1,828$ \\
\hline & & & $\begin{array}{l}\text { End treatment- } 12 \text { months } \\
\text { post-treatment (total costs): }\end{array}$ & $€ 452(S E=14)$ \\
\hline & \multirow[b]{2}{*}{$\begin{array}{l}\text { Effects intervention } \\
\text { CBCL: Externalizing scores, } \\
\text { internalizing scores: } \\
\text { GSI: Global severity index are measures } \\
\text { The main effects were not showed } \\
\text { in this study but only differences over } \\
\text { time were presented. }\end{array}$} & \multirow[b]{2}{*}{$\begin{array}{l}\text { Effects comparator } \\
\text { CBCL: Externalizing scores, } \\
\text { internalizing scores: } \\
\text { GSI: Global severity index } \\
\text { The main effects were not showed in this } \\
\text { study but only differences over time were } \\
\text { presented. }\end{array}$} & \multicolumn{2}{|c|}{ Difference effects (Effects ${ }_{C A U}$-Effects MST $_{\text {) }}$ (after risk adjusted model): } \\
\hline & & & $\begin{array}{l}\text { 0-end treatment: } \\
\text { end treatment- } 12 \text { months } \\
\text { post-treatment: }\end{array}$ & $\begin{array}{l}\text { Externalizing:-14.75 (SE }=8.37) \\
\text { Internalizing:-14.19 }(\mathrm{SE}=9.26) \\
\text { Global severity index: }-0.03 \\
(\mathrm{SE}=0.497) \\
\text { Externalizing:3.29 }(\mathrm{SE}=9.97) \\
\text { Internalizing:-6.18 }(\mathrm{SE}=9.67) \\
\text { Global severity index: }-0.37 \\
(\mathrm{SE}=0.428)\end{array}$ \\
\hline
\end{tabular}

Results ICER: 1 point improvement in externalizing scores for usual care was associated with a cost of $€ 1,561.1$ point improvement in externalizing scores for MST was associated with a costs of $€ 404$. After 12 months both treatments have comparable costs and externalizing scores. Studies considering costs and benefits

$\begin{array}{llc}\text { Olsson }^{4} & \text { Costs intervention (MST) } & \text { Costs comparator (CAU) } \\ (2010)[26] & \text { Treatment costs: } € 10.789 & \text { Travel: } € 151 \text { (225) } \\ & \text { Travel: } € 53(133) & \end{array}$

Results The net loss to society after two years is $€ 4.555$

$\begin{array}{ll}\text { Cary } & \text { Costs interventions (MST + YOT) } \\ & \text { Treatment costs: } € 3.013(1.940) \\ & \text { Social worker: } € 733(446) \\ & \text { Reparation worker: } € 100(131) \\ & \text { Drugs worker: } € 54(74) \\ & \text { Connexions worker: } € 33(69) \\ & \text { Parenting worker: } € 36(137)\end{array}$
Costs comparator (YOT) Social worker: $€ 1.023$ (779) Reparation worker: $€ 83$ (14) Drugs worker: $€ 78$ (152) Connexions worker: $€ 18$ (61) Parenting worker: $€ 36(137) \quad$ Group worker: $€ 22$ (44)
Benefits intervention (MST) Psychosocial and behavioral effects: - $\quad$ Program effects Social services (placement): $\quad$ Social services (Placement): $€ 31.947$ (€65.869) $\quad € 36.707$ (73.407) Social services (nonplacement): $\quad$ Social services (nonplacement): $€ 8.557$ (19.459)
National board of institutional care (rebate): $€ 3.009$ (11.014) National board of institutional care (placements): $€ 3.593$ (31.937) Wider societal costs and benefit: set to zero Psychosocial and behavioral effects: $€ 14.914$ (15.405) National board of institutional care (rebate): € 2.375 (9.949) National board of institutional care (placements): 0 (0) SEK Wider societal costs and benefit: set to zero set to zero

Benefits interventions (MST + YOT)

Benefits comparator (YOT) Offending behavior (Young offender information system):
$€ 12,397$ (18 472)
$€ 15,409(24,013)$ 
Table 4 Studies considering externalizing disorders and delinquency (Continued)

\begin{tabular}{|c|c|c|c|c|c|}
\hline \multirow{4}{*}{\multicolumn{2}{|c|}{$\begin{array}{l}\text { Dopp } \\
\text { (2014) [30] }\end{array}$}} & $\begin{array}{l}\text { Group worker: } € 17 \text { (34) } \\
\text { Psychologist: } € 17(67) \\
\text { Other appointments: } € 20 \text { (59) }\end{array}$ & $\begin{array}{l}\text { Psychologist: } € 30 \text { (91) } \\
\text { Other appointments: } € 26(95)\end{array}$ & & \multirow[b]{4}{*}{$\begin{array}{l}\text { Benefits comparator (IT) } \\
\text { Benefits for taxpayer } \\
\text { Murder: } € 0 \\
\text { Sexual offenses: } € 602 \\
\text { Robbery: } € 308 \\
\text { Assault: } € 1.697 \\
\text { Property: } € 1.899 \\
\text { Drug: } € 1.334 \\
\text { Theft: } € 188 \\
\text { Stolen property: } € 53 \\
\text { Fraud: } € 224 \\
\text { Assault: } € 294 \\
\text { Drug: } € 598 \\
\text { TOTAL: } € 7.197\end{array}$} \\
\hline & & \multicolumn{3}{|c|}{$\begin{array}{l}\text { Difference (Costs + benefits) between treatments } € 1.612 \text { (95\% C.I- } € 7.699-€ \text { to } 10.924) \\
\text { In the cost-effectiveness plane, we see, there is } 63 \% \text { probability that the net benefit of MST + Yot is positive in favor of the MST + YOT group. }\end{array}$} & \\
\hline & & \multirow{2}{*}{$\begin{array}{l}\text { Costs interventions (MST) } \\
\text { Costs per patient: } € 9,756\end{array}$} & \multirow{2}{*}{$\begin{array}{l}\text { Costs comparator (CAU) } \\
\text { Costs per patient: } € 1,843\end{array}$} & Benefits intervention (MST) & \\
\hline & & & & $\begin{array}{l}\text { Benefits for taxpayer } \\
\text { Murder: } € 0 \\
\text { Sexual offenses: } € 922 \\
\text { Robbery: } € 188 \\
\text { Assault: } € 1.156 \\
\text { Property: } € 2.395 \\
\text { Drug: } € 916 \\
\text { Theft: } € 131 \\
\text { Stolen property: } € 24 \\
\text { Fraud: } € 259 \\
\text { Assault: } € 236 \\
\text { Drug: } € 777 \\
\text { TOTAL: } € 7.007\end{array}$ & \\
\hline & Results & $\begin{array}{l}\text { Crime victim avoided expenses } \\
\text { Murder/manslaughter } \\
\text { Tangible: } € 6.125 \\
\text { Intangible: } € 11.365 \\
\text { Sexual } \\
\text { Tangible: } € 259 \\
\text { Intangible: } € 3.439 \\
\text { Robbery } \\
\text { Tangible: } € 575 \\
\text { Intangible: } € 1.422 \\
\text { Assault } \\
\text { Tangible: } € 539 \\
\text { Intangible: } € 2.926 \\
\text { Property } \\
\text { Tangible: } € 3.914 \\
\text { Intangible: } € 0 \\
\text { Drug } \\
\text { Tangible: } € 0 \\
\text { Intangible: } € 0 \\
\text { TOTAL } \\
\text { Tangible: } € 11.412 \\
\text { Intangible: } € 19.151\end{array}$ & $\begin{array}{l}\text { Net present values and benefit-cost ratios } \\
\text { Net present value } \\
\text { Referred youths } \\
\text { Taxpayer: } € 2.348 \\
\text { Crime victim tangible: } € 2.389 \\
\text { Crime victim intangible } € 9.375 \\
\text { Cumulative: } € 29.939 \\
\text { Siblings: } \\
\text { Taxpayer: } € 674 \\
\text { Crime victim tangible: } € 2.702 \\
\text { Crime victim intangible: } € 4.533 \\
\text { Cumulative: } € 6.561 \\
\text { Sibling pairs } \\
\text { Taxpayer: } € 1.399 \\
\text { Crime victim tangible: } € 3.499 \\
\text { Crime victim intangible: } € 11.238 \\
\text { Cumulative*: } € 31.962 \\
\text { *: Includes the incremental costs of } \\
\text { MST over IT } \\
\text { Benefit cost ratio } \\
\text { Referred youths } \\
\text { Taxpayer: } 1.3 \\
\text { Crime victim tangible: } 1.3 \\
\text { Crime victim intangible } 2.19 \\
\text { Cumulative: } 4.78 \\
\text { Siblings: } \\
\text { Taxpayer: - } \\
\text { Crime victim tangible: - } \\
\text { Crime victim intangible: - } \\
\text { Cumulative: - }\end{array}$ & $\begin{array}{l}\text { Sensitivity analysis } \\
\text { Max (plausible) values } \\
\text { Crime victim intangible benefits: } € 48.087 \\
\text { Sibling juvenile arrest rates: } € 30.74 \\
\text { Discount rates: } € 24.063 \\
\text { Min (plausible) values } \\
\text { Crime victim intangible benefits: } € 17.561 \\
\text { Sibling juvenile arrest rates:- } \\
\text { Discount rates: } € 36.704\end{array}$ & \\
\hline
\end{tabular}

intangible benefits: $€ 48087$

ile arrest rates: $€ 30.74$

ount rates: $€ 24.063$

Crime victim intangible benefits: $€ 17.561$

Sibling juvenile arrest rates:

Siblings:

Crime victim tangible: $€ 2.702$

Sibling pairs

ayer: $€ 1.399$

cumulative*: € 31.962

cumulative: 4.78

Taxpayer: -

rime victim intangible:

Cumulative: - 
Table 4 Studies considering externalizing disorders and delinquency (Continued)

\begin{tabular}{|c|c|c|c|c|}
\hline & & $\begin{array}{l}\text { Sibling pairs } \\
\text { Taxpayer: } 1.18 \\
\text { Crime victim tangible: } 1.44 \\
\text { Crime victim intangible: } 2.42 \\
\text { Cumulative*: } 5.04 \\
\text { *: Includes the incremental costs } \\
\text { of MST over CAU }\end{array}$ & & \\
\hline \multirow[t]{2}{*}{$\begin{array}{l}\text { Borduin } \\
\text { (2015) [31] }\end{array}$} & $\begin{array}{l}\text { Costs interventions (MST-PSB) } \\
\text { Costs per patient: } € 10,566\end{array}$ & $\begin{array}{l}\text { Costs comparator (CAU) } \\
\text { Costs per patient: } € 4,610\end{array}$ & $\begin{array}{l}\text { Benefits intervention (MST-PSB) } \\
\text { Benefits for taxpayer } \\
\text { Murder: } € 0 \\
\text { Sexual offenses: } € 6.419 \\
\text { Robbery: } € 2.189 \\
\text { Assault: } € 0 \\
\text { Property: } € 2.831 \\
\text { Drug: } € 1.899 \\
\text { Theft: } € 180 \\
\text { Stolen property } € 0 \\
\text { Fraud: } € 91 \\
\text { Assault: } € 250 \\
\text { Drug: } € 512 \\
\text { TOTAL: } € 14.371\end{array}$ & $\begin{array}{l}\text { Benefits comparator (CAU) } \\
\text { Benefits for taxpayer } \\
\text { Murder: } € 0 \\
\text { Sexual offenses: } € 15.756 \\
\text { Robbery: } € 0 \\
\text { Assault: } € 2.194 \\
\text { Property: } € 3.790 \\
\text { Drug: } € 518 \\
\text { Theft: } € 65 \\
\text { Stolen property: } € 39 \\
\text { Fraud: } € 75 \\
\text { Assault: } € 289 \\
\text { Drug: } € 112 \\
\text { TOTAL: } € 22.839\end{array}$ \\
\hline & $\begin{array}{l}\text { Crime victim avoided expenses } \\
\text { Murder/manslaughter } \\
\text { Tangible: } € 41.048 \\
\text { Intangible: } € 76.169 \\
\text { Sexual } \\
\text { Tangible: } € 1.739 \\
\text { Intangible: } € 23.044 \\
\text { Robbery } \\
\text { Tangible: } € 3.850 \\
\text { Intangible: } € 9.529 \\
\text { Assault } \\
\text { Tangible: } € 3.612 \\
\text { Intangible: } € 19.611 \\
\text { Property } \\
\text { Tangible: } € 26.244 \\
\text { Intangible: } € 0 \\
\text { TOTAL } \\
\text { Tangible: } € 76.494 \\
\text { Intangible: } € 128.353\end{array}$ & $\begin{array}{l}\text { Net present values and benefit-cost ratios } \\
\text { Net present value } \\
\text { Referred youths } \\
\text { Taxpayer: } € € 79.891 \\
\text { Crime victim tangible: } € 70.538 \\
\text { Crime victim intangible } € 122.397 \\
\text { Cumulative*: } € 284.739 \\
\text { Siblings: } \\
\text { Benefit cost ratio } \\
\text { Referred youths } \\
\text { Taxpayer: } 14.41 \\
\text { Crime victim tangible: } 12.84 \\
\text { Crime victim intangible } 21.55 \\
\text { Cumulative: } 48.81 \\
\text { *: Includes the incremental costs of } \\
\text { MST over CAU }\end{array}$ & $\begin{array}{l}\text { Sensitivity analysis } \\
\text { Max (plausible) values } \\
\text { Crime victim intangible benefits: } € 387.085 \\
\text { Discount rates: } € 239.009 \\
\text { Posttreatment arrest rates: } € 478.277 \\
\text { Min (plausible) values } \\
\text { Crime victim intangible benefits : } € 188.217 \\
\text { Discount rates: } € 311.107 \\
\text { Posttreatment arrest rates: } € 91.673\end{array}$ & \\
\hline
\end{tabular}
For Schoenwald et al. (2006), 1996 was taken as prices year although the study was also published in 1996. This was because they already published their first study in 1996 (preliminary findings) and subsequently probably the current study was conducted in 1996

MST multisystemic therapy, Joint combination of individual and family therapy, group skill-focused psycho-education group intervention, CAU care as usual, FSN family support network, MDFT multidimensional family treatment, MET/CBT12 motivational enhancement treatment/cognitive behavior therapy, 12 sessions, MET/CBT5 motivational enhancement treatment/cognitive behavior therapy, 5 sessions, ACRA adolescent community reinforcement approach, $D C$ drug court with community services, $D C+M S T$ drug court with multisystemic therapy; $D C+M S T+C M$ drug court with MST and enhanced with a contingency management programs, FFT functional family therapy, FC family court with community services, MST-PSB MST for sexual behaviors; ICER incremental cost-effectiveness ratio 
Studies considering costs and benefits Three of the studies that considered adolescents with substance use disorders, considered costs and benefits [22, 24, 32]. The study of French et al. [22] indicated that MET/CBT-5, MET/CBT-12 and FSN generated significant economic benefits to society for substance abusing adolescents, MDFT and ACRA did not generate these benefits. MCcollister et al. [24] showed that the savings in costs offset the treatment costs of DC, especially for DC/MST/ $\mathrm{CM}$, in juvenile drug court participants when compared to FC (Family court with community services). Schoenwald [32] showed that the monetary benefits of MST compared to CAU for substance use disorder almost offset the higher costs of MDFT. Over time the difference between benefits and costs may be reduced to a complete offset.

\section{Delinquency/externalizing disorders}

Five studies considered adolescents with delinquency or externalizing disorders; the study of Sheidow et al. [23], Olsson [26], Cary et al. [28], Dopp et al. [30] and Borduin et al. [31] respectively included patients with a psychiatric crisis, patients with a conduct disorder,delinquent adolescents, serious juvenile offenders and juvenile sexual offenders. One study, Sheidow et al. [23], considered both costs and effects and four studies [26, 28, 30,31] considered both costs and benefits.

Studies considering costs and effects In the study of Sheidow et al. [23], MST was effective in the short term (4 months) in terms of externalizing behavior compared to care as usual for patients with psychiatric emergencies. But MST appeared equally effective on the cost measure over the long term (12 months).

Studies considering costs and benefits Olsson [26] showed that for adolescents with conduct disorder MST's benefits did not offset the costs and that MST was subsequently associated with a net loss to society. The study of Cary et al. [28] showed that MST in combination with CAU has a scope to generate cost savings when compared to providing CAU alone. The costbenefit study of Dopp et al. [30] indicated that MST, when delivered to serious juvenile offenders, produces economic benefits well into adulthood. Borduin et al. [31] showed that when juvenile sexual offenders are treated with MST-PSB; this treatment can produce lasting economic benefits.

\section{Quality of the studies}

Only for one study [23] commentary was available from the NHS-EED. We compared the commentary on the study with our quality assessment checklists to evaluate if all issues were addressed. The quality of the studies was not only assessed for the 7 unique studies but for the 9 studies. The argument for including all studies was to differentiate between methods (e.g. analysis), display of results and discussion even though they were based on the same study. The quality assessed with the BMJ checklist was between 52 and $86 \%$ (Table 5 ). The quality assessed with the CHEC list was between 50 and $79 \%$ (Table 5). Up to date, there are no thresholds (minimum number of criteria satisfied) for these checklists to determine the difference between bad and good quality economic evaluations [18]. Overall, the outcomes on the checklists matched although quality assessed with the CHEC list was consequently lower. The largest difference in quality percentages was $20 \%$. All studies clearly stated their primary outcome measures. Most studies did not report all relevant costs and effects.

\section{Risk of bias}

All studies were RCTs [22-32]. Two of these studies [23, 32] only included patients receiving Medicaid (an aid program regarding insurances for low income families in the United States). For these studies, the RCT of the effect study contained (due to randomization) balanced samples. However, these samples were not checked for balance after the selection of participants who received medicaid, so they were at risk for selection bias. All studies had a high risk of performance bias, as blinding of both therapist and patient is impossible. For two studies [23, 32] blinding was not necessary as both the cost and outcome data were extracted from existing data systems (The medicaid billing records). Although blinding of outcome assessors is possible to reduce detection bias, no study reported to have done so. Blinding is also necessary for pre-allocation assessment. All studies were based on randomized controlled trials where allocation concealment is necessary. The studies included in this review, did not explicitly refer to the allocation concealment. Three studies were at risk of attrition bias. These three studies did not describe the number of patients that dropped out from the study [24, 27, 32]. Two studies only described the overall attrition rate [22, 25]. For one study [29] however, overall attrition rate could be extracted by using the study of French et al. [22] as it was based on the same participants. Dropout in the effect-study of Sheidow et al. [23] was low and although no dropout was described for the economic evaluation, as the economic evaluation is based on the same participants, this is expected to be low. Overall, dropout rate (when measured) seemed low. Reporting bias was assessed by reading protocols from the studies and no bias was reported. Only for two studies $[22,29]$ a protocol existed. Other studies did not have such a protocol, although for three studies trial registrations were present $[24,27,28]$. There were no indications of deviations from the original design. The economic evaluations did not always include all clinical 
Table 5 Assessments of the quality of the studies with the Drummond checklist and the CHEC list

\begin{tabular}{|c|c|c|c|c|c|c|c|c|c|c|c|}
\hline British Medical Journal Checklist & $1^{\text {a }}$ & $2^{a}$ & $3^{a}$ & $4^{a}$ & $5^{a}$ & $6^{a}$ & $7^{a}$ & $8^{\mathrm{a}}$ & $9^{a}$ & $10^{\mathrm{a}}$ & $11^{\mathrm{a}}$ \\
\hline 1. The research question is stated. & - & - & $\checkmark$ & $\checkmark$ & $\checkmark$ & $\checkmark$ & $\checkmark$ & - & $\checkmark$ & $\checkmark$ & $\checkmark$ \\
\hline 2. The economic importance of the research question is stated. & $\checkmark$ & - & $\checkmark$ & $\checkmark$ & - & $\checkmark$ & $\checkmark$ & $\checkmark$ & $\checkmark$ & $\checkmark$ & $\checkmark$ \\
\hline 3. The viewpoint(s) of the analysis are clearly stated and justified. & - & $\checkmark$ & - & $\checkmark$ & - & - & $\checkmark$ & $\checkmark$ & - & - & - \\
\hline $\begin{array}{l}\text { 4. The rationale for choosing alternative programmes or } \\
\text { interventions compared is stated. }\end{array}$ & $\checkmark$ & - & - & - & - & - & $\checkmark$ & - & $\checkmark$ & - & - \\
\hline 5. The alternatives being compared are clearly described & $\checkmark$ & $\checkmark$ & $\checkmark$ & $\checkmark$ & - & - & - & - & $\checkmark$ & $\checkmark$ & $\checkmark$ \\
\hline 6. The form of economic evaluation used is stated. & - & $\checkmark$ & $\checkmark$ & $\checkmark$ & - & $\checkmark$ & $\checkmark$ & $\checkmark$ & $\checkmark$ & $\checkmark$ & $\checkmark$ \\
\hline $\begin{array}{l}\text { 7. The choice of form of economic evaluation is justified in } \\
\text { relation to the questions addressed. }\end{array}$ & NC & $\checkmark$ & $\checkmark$ & $\checkmark$ & - & $\checkmark$ & $\checkmark$ & $\checkmark$ & $\checkmark$ & $\checkmark$ & $\checkmark$ \\
\hline 8. The source(s) of effectiveness estimates used are stated. & $\checkmark$ & $\checkmark$ & $\checkmark$ & $\checkmark$ & $\checkmark$ & $\checkmark$ & $\checkmark$ & $\checkmark$ & $\checkmark$ & $\checkmark$ & $\checkmark$ \\
\hline $\begin{array}{l}\text { 9. Details of the design and results of effectiveness study are } \\
\text { given (if based on a single study). }\end{array}$ & $\checkmark$ & NA & $\checkmark$ & $\checkmark$ & $\checkmark$ & $\checkmark$ & $\checkmark$ & $\checkmark$ & - & $\checkmark$ & $\checkmark$ \\
\hline $\begin{array}{l}\text { 10. Details of the methods of synthesis or meta-analysis of } \\
\text { estimates are given (if based on a synthesis of a number of } \\
\text { effectiveness studies). }\end{array}$ & NA & NA & NA & NA & NA & NA & NA & NA & NA & NA & NA \\
\hline $\begin{array}{l}\text { 11. The primary outcome measure(s) for the economic } \\
\text { evaluation are clearly stated. }\end{array}$ & $\checkmark$ & $\checkmark$ & $\checkmark$ & $\checkmark$ & $\checkmark$ & $\checkmark$ & $\checkmark$ & $\checkmark$ & $\checkmark$ & $\checkmark$ & $\checkmark$ \\
\hline 12. Methods to value benefits are stated. & $\checkmark$ & $\checkmark$ & NA & $\checkmark$ & $\checkmark$ & NA & $\checkmark$ & NA & $\checkmark$ & $\checkmark$ & $\checkmark$ \\
\hline $\begin{array}{l}\text { 13. Details of the subjects from whom valuations were } \\
\text { obtained were given. }\end{array}$ & $\checkmark$ & $\checkmark$ & $\checkmark$ & $\checkmark$ & $\checkmark$ & $\checkmark$ & $\checkmark$ & $\checkmark$ & $\checkmark$ & $\checkmark$ & $\checkmark$ \\
\hline 14. Productivity changes (if included) are reported separately. & NA & $\checkmark$ & NA & NA & NA & NA & NA & NA & NA & - & - \\
\hline $\begin{array}{l}\text { 15. The relevance of productivity changes to the study } \\
\text { question is discussed. }\end{array}$ & - & - & - & - & - & - & $\checkmark$ & - & - & - & - \\
\hline $\begin{array}{l}\text { 16. Quantities of resource use are reported separately from } \\
\text { their unit costs. }\end{array}$ & $\checkmark$ & $\checkmark$ & - & - & - & - & - & - & $\checkmark$ & $\checkmark$ & $\checkmark$ \\
\hline $\begin{array}{l}\text { 17. Methods for the estimation of quantities and unit costs } \\
\text { are described. }\end{array}$ & - & - & - & - & $\checkmark$ & - & $\checkmark$ & $\checkmark$ & $\checkmark$ & $\checkmark$ & $\checkmark$ \\
\hline 18. Currency and price data are recorded. & $\checkmark$ & $\checkmark$ & - & $\checkmark$ & - & - & - & $\checkmark$ & $\checkmark$ & $\checkmark$ & $\checkmark$ \\
\hline $\begin{array}{l}\text { 19. Details of currency of price adjustments for inflation or } \\
\text { currency conversion are given. }\end{array}$ & $\checkmark$ & $\checkmark$ & - & - & - & - & $\checkmark$ & - & $\checkmark$ & $\checkmark$ & $\checkmark$ \\
\hline 20. Details of any model used are given & NA & $\checkmark$ & $\checkmark$ & $\checkmark$ & $\checkmark$ & $\checkmark$ & NA & NA & $\checkmark$ & NA & NA \\
\hline $\begin{array}{l}\text { 21. The choice of model used and the key parameters on } \\
\text { which it is based are justified. }\end{array}$ & NA & - & - & $\checkmark$ & - & - & NA & NA & - & NA & NA \\
\hline 22. Time horizon of costs and benefits is stated. & $\checkmark$ & $\checkmark$ & $\checkmark$ & $\checkmark$ & $\checkmark$ & $\checkmark$ & $\checkmark$ & $\checkmark$ & $\checkmark$ & $\checkmark$ & $\checkmark$ \\
\hline 23. The discount rate(s) is stated. & NA & NA & NA & NA & NA & NA & $\checkmark$ & NA & $\checkmark$ & $\checkmark$ & $\checkmark$ \\
\hline 24. The choice of discount rate(s) is justified. & NA & NA & NA & NA & NA & NA & $\checkmark$ & NA & $\checkmark$ & $\checkmark$ & $\checkmark$ \\
\hline $\begin{array}{l}\text { 25. An explanation is given if costs and benefits are not } \\
\text { discounted. }\end{array}$ & NA & NA & NA & NA & NA & NA & NA & NA & NA & NA & NA \\
\hline $\begin{array}{l}\text { 26. Details of statistical tests and confidence intervals are } \\
\text { given for stochastic data. }\end{array}$ & - & - & $\checkmark$ & - & $\checkmark$ & - & $\checkmark$ & $\checkmark$ & $\checkmark$ & - & - \\
\hline 27. The approach to sensitivity analysis is given. & $\checkmark$ & - & $\checkmark$ & - & - & - & $\checkmark$ & NC & $\checkmark$ & $\checkmark$ & $\checkmark$ \\
\hline 28. The choice of variables for sensitivity analysis is justified. & $\checkmark$ & NA & NA & NA & NA & NA & $\checkmark$ & NA & $\checkmark$ & $\checkmark$ & $\checkmark$ \\
\hline 29. The ranges over which the variables are varied are justified. & NC & NA & NA & NA & NA & NA & $\checkmark$ & NA & $\checkmark$ & $\checkmark$ & $\checkmark$ \\
\hline 30. Relevant alternatives are compared. & $\checkmark$ & NC & - & NC & $\checkmark$ & NS & $\checkmark$ & $\checkmark$ & $\checkmark$ & $\checkmark$ & $\checkmark$ \\
\hline 31. Incremental analysis is reported. & $\checkmark$ & $\checkmark$ & - & $\checkmark$ & $\checkmark$ & - & $\checkmark$ & - & $\checkmark$ & $\checkmark$ & $\checkmark$ \\
\hline $\begin{array}{l}\text { 32. Major outcomes are presented in a disaggregated as } \\
\text { well as aggregated form }\end{array}$ & $\checkmark$ & $\checkmark$ & $\checkmark$ & - & $\checkmark$ & $\checkmark$ & $\checkmark$ & $\checkmark$ & $\checkmark$ & $\checkmark$ & $\checkmark$ \\
\hline 33. The answer to the study question is given. & $\checkmark$ & NC & $\checkmark$ & $\checkmark$ & $\checkmark$ & $\checkmark$ & $\checkmark$ & $\checkmark$ & $\checkmark$ & $\checkmark$ & $\checkmark$ \\
\hline 34. Conclusions follow from the data reported. & $\checkmark$ & $\checkmark$ & $\checkmark$ & $\checkmark$ & $\checkmark$ & $\checkmark$ & $\checkmark$ & $\checkmark$ & $\checkmark$ & - & - \\
\hline
\end{tabular}


Table 5 Assessments of the quality of the studies with the Drummond checklist and the CHEC list (Continued)

35. Conclusions are accompanied by the appropriate caveats. Total score British medical journal checklist

\section{CHEC list}

1. Is the study population clearly described?

2. Are competing alternatives clearly described?

3. Is a well-defined research question posed in answerable form?

4. Is the economic study design appropriate to the stated objective?

5. Is the chosen time horizon appropriate to include relevant costs and consequences?

6. Is the actual perspective chosen appropriate?

7. Are all important and relevant costs for each alternative identified?

8. Are all costs measured appropriately in physical units?

9. Are costs valued appropriately?

10. Are all important and relevant outcomes for each alternative identified?

11. Are all outcomes measured appropriately?

12. Are outcomes valued appropriately?

13. Is an incremental analysis of costs and outcomes of alternatives performed?

14. Are all future costs and outcomes discounted appropriately?

15. Are all important variables, whose values are uncertain, appropriately subjected to sensitivity analysis?

16. Do the conclusions follow from the data reported?

17. Does the study discuss the generalizability of the results to other settings and patient/client groups?

18. Does the article indicate that there is no potential conflict of interest of study researcher(s) and funder(s)?

19. Are ethical and distributional issues discussed appropriately?

Total score $\mathrm{CHEC}^{\mathrm{b}}$

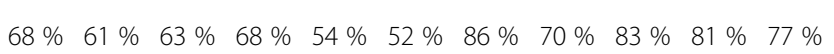

$\begin{array}{lllllllllll}\checkmark & \checkmark & \checkmark & \checkmark & \checkmark & \checkmark & \checkmark & \checkmark & \checkmark & \checkmark & \checkmark \\ \checkmark & \checkmark & \checkmark & \checkmark & - & - & - & - & \checkmark & \checkmark & \checkmark \\ - & - & \checkmark & \checkmark & \checkmark & \checkmark & \checkmark & - & \checkmark & \checkmark & \checkmark \\ \checkmark & \checkmark & \checkmark & \checkmark & \checkmark & \checkmark & \checkmark & \checkmark & \checkmark & \checkmark & \checkmark \\ \text { NS } & \text { NS } & \checkmark & \text { NS } & \text { NS } & \text { NS } & \checkmark & \text { NS } & \text { NS } & \checkmark & \checkmark\end{array}$

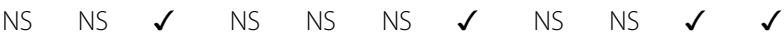

$\begin{array}{lllllllllll}- & \checkmark & - & - & - & - & \checkmark & - & - & - & - \\ - & - & N S & - & - & - & - & - & - & \checkmark & \checkmark\end{array}$

$\begin{array}{lllllllllll} & & & & & & & & & & \\ \checkmark & \checkmark & - & \checkmark & \checkmark & \text { NS } & \checkmark & \checkmark & \checkmark & \checkmark & \checkmark \\ - & - & \checkmark & \checkmark & - & \checkmark & \checkmark & \checkmark & \checkmark & \checkmark & \checkmark\end{array}$

$\begin{array}{ccccccccccc} & & \checkmark & \checkmark & - & \checkmark & \checkmark & \checkmark & \checkmark & \checkmark & \checkmark \\ \checkmark & \checkmark & \checkmark & \checkmark & \checkmark & \checkmark & \checkmark & \checkmark & \checkmark & \checkmark & \checkmark \\ - & \checkmark & \checkmark & \checkmark & \checkmark & \checkmark & - & \checkmark & \checkmark & \checkmark & \checkmark \\ \checkmark & \checkmark & - & \checkmark & \checkmark & - & \checkmark & - & \checkmark & \checkmark & \checkmark\end{array}$

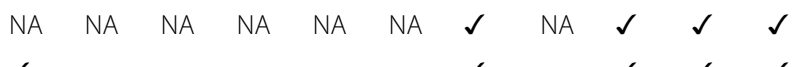

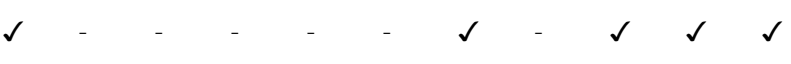

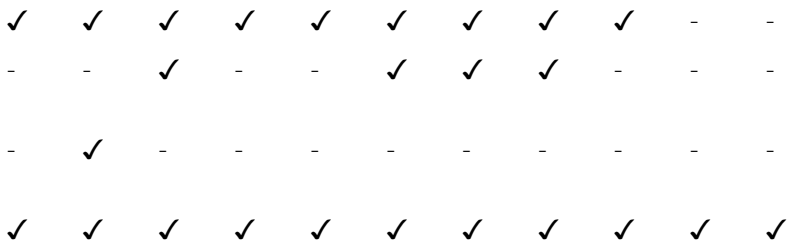

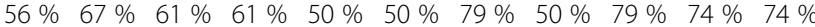

NS not stated, NA not applicable, NC not clear

Explanation criteria checklist: British medical journal checklist: 1. A specific question is not necessary, as long as the goal of the research is clearly stated; 5 . The competing alternatives may also be described in a different accessible paper from the RCT in more detail 10. The presentation of the results is clearly given and discussions of the study contain generalizability and comparison with other studies. CHEC list: 5: Chosen time horizon is appropriate when after a certain time no additional effects are attained

${ }^{a}$ Studies: Schoenwald et al., 1996; 2 French et al., 2003; 3 Sheidow et al., 2004; 4 Dennis et al., 2004; 5 McCollister et al., 2009; 6 French et al., 2008; 7 Olsson, 2010; 8 Sheidow et al., 2012; 9 Cary et al., 2013; 10 Dopp et al., 2014; 11. Borduin et al., 2015

${ }^{\mathrm{b}} \mathrm{S}$ cores were calculated by dividing the positively checked items on the quality checklist by the total minus items on the checklist that were not applicable (NA) to the study

outcomes that were available [23-26, 32] as there was often only interest in specific outcomes. One study [25] excluded clinical outcomes as there was no difference between treatments in terms of outcomes and so only costs were considered (costs minimization). The exclusion of outcomes was not related to possible negative impact on the results as effects in the studies were equally or more beneficial when compared to the effects of the comparator.

\section{Methodological summary}

Uncertainty around treatment costs was not presented in four studies as averages of these costs were used [24, 27, $30,31]$. In six studies [22, 23, 25, 29-32] uncertainty around the (other) estimates was not (fully) addressed. In seven studies, a simple one way sensitivity analysis was used to assess the impact that changes in a certain parameter will have on the conclusions [22, 23, 26, 28, 30-32]. In two studies, sensitivity analysis was applied by imputing missing data in different ways. Outcomes proved to be robust [27, 28]. Two studies performed scenario analyses meaning that cost estimates (surrounded by uncertainty) were increased or decreased. Data proved to be robust [26, 32]. In another study a sensitivity analysis was carried out to assess the effect which outliers in each therapy group had on outcomes, but this did not have an effect the results. In the studies of Dopp et al. [31] and 
Borduin et al. [31] a sensitivity analysis was applied by using plausible minimum and maximum values (obtained from other studies) for offense categories, arrest rates and discount rates. French et al. [22] used different models which assessed the effect on using more or less covariates in the models but it did not affect the results. In six of the studies cost-effectiveness/utility/benefits were assessed based on models [22-25, 28, 32]. Four of these studies used simple regression models [23-25, 28] and two used a more advanced least squares random effect model $[19,26]$. The remaining three studies did not integrate any model in the analysis. Three studies did not report their price year (the year to which costs are indexed) $[23,24,32]$.

Authors of three studies indicated that a societal perspective was adopted, where not only health care costs but also other costs, for example those associated with lost or impaired ability to work, were taken into account $[22,26,29]$. However, this was only true for the study of Olsson [26], as this was the only study to assess costs outside the health care sector. In the studies of Dennis et al. [29] and French et al. [22], the societal part was defined as using market values for calculating the costs of goods and services used. Dopp et al. [30] and Borduin et al. [31] conducted cost-benefit analyses and did not explicitely mention their perspective. Both studies focused on taxpayer benefits and expressed intagible benefits in monetary values. Cary et al. [28] used a narrow perspective as only services that were recorded by a specific data-system were included (appointments with social workers, connexion workers (a United Kingdom (UK) governmental information, advice, guidance and support service for young people aged thirteen to nineteen), reparation workers (coordinates and supports a range of interventions and community reparation projects that young people will have to undertake as part of their Referral or Community Order), parenting workers, group workers and psychologists). Sheidow [23] adopted the perspective of an institution. Other studies did not explicitly state their perspective. Most of the studies only reported treatment costs. A summary of the costs and clinical outcomes measured in the studies is provided in Table 6 .

Following Drummond et al. [33], full economic evaluations should not only report costs, but also health outcomes. Four studies were classified as cost-effectiveness analyses [23, 25, 27, 29]. Only one of these studies compared treatments using an incremental cost-effectiveness ratio [29] as described for instance by Drummond et al. [33]. The cost-effectiveness analysis of French et al. [25] was reduced to a simple cost minimization analysis as the effects of both treatments after analysis proved to be similar. Sheidow et al. [27] calculated average costeffectiveness ratios (ACER), which means that there was no direct comparison between treatments but only between the before- and after treatment costs and effects of every participant. In four studies it was explicitly stated that cost-benefit analyses [22, 26, 30, 31] were performed. Olsson [26] considered psychosocial and behavioral effects, but as no difference was observed regarding these clinical measures between treatments, these effects were excluded from the analysis. French et al. [22] did not value the health outcomes on which the intervention was focused (like reduction in days of substance use) but did value the effects of treatment on education, employment and criminal activity. Dopp et al. [30] and Borduin et al. [31] conducted a cost-benefit analysis; the cost outcome were the treatment costs and the benefits were defined as taypayer benefits, tangible benefits and intangible benefits were expressed in monetary values. Cary et al. [28] classified his study as a cost-offset evaluation. He calculated the net-benefit, but stated that his study cannot be viewed as a costeffectiveness study as he did not measure health outcome. Two studies did not state the type of economic analyses they performed [24, 32], but did consider both costs and benefits. Mcollister [24] indicated that her study was not a full economic evaluation, as she only considered treatment costs. This is also the case concerning the study of Sheidow et al. [27], however, this study was stated to be a cost-effectiveness analysis. Furthermore, Schoenwald et al. [32] did not classify their study explicitly but considered both costs of different health care services and monetary benefits so it can be considered a cost-benefit analysis.

\section{Limitation/generalizability summary}

Four studies commented on their generalizability [23, 25-27]. Sheidow et al. [23] reported that as their sample only consisted of youths enrolled in Medicaid, which are generally economically less advantaged, findings cannot be generalized to a more economically advantaged population. The same is true, although not stated, for the study of Schoenwald et al. [32] who also analyzed Medicaid data. The study of Olsson [26] was conducted in Sweden, where MST is twice more expensive than in the USA and may play a different role in society. MST in Sweden may be used as an alternative to nonplacement interventions as opposed to an alternative to placement interventions as found in other studies. Also in the study of French et al. [25], which was conducted in Mexico, location and small sample size were indicated as limitations for generalizability. The same was true, although not stated, for the study of Cary et al. [28] which was conducted in the United Kingdom. Also an important limitation (but not mentioned as such) were the omissions of uncertainty around the estimates in the studies of Dopp et al. [30] and Borduin et al. [31], so the results 
Table 6 Overview of costs and clinical outcome measures used in studies

\begin{tabular}{|c|c|c|c|c|c|}
\hline & Treatment costs & $\begin{array}{l}\text { Other healthcare } \\
\text { costs }\end{array}$ & $\begin{array}{l}\text { Costs outside } \\
\text { health care sector }\end{array}$ & $\begin{array}{l}\text { Perspective used in } \\
\text { the economic evaluations }\end{array}$ & Clinical outcome measure \\
\hline (Schoenwald et al., 1996) [32] & $\checkmark$ & $\checkmark$ & & Healthcare & - \\
\hline (French et al., 2003) [22] & $\checkmark$ & & & Institution & - \\
\hline (Sheidow et al., 2004) [23] & $\checkmark$ & $\checkmark$ & & Healthcare & $\mathrm{CBCL} / \mathrm{GSI}$ \\
\hline (Dennis et al., 2004) [29] & $\checkmark$ & & & Institution & - \\
\hline (McCollister et al., 2009) [24] & $\checkmark$ & & & Institution & SRD \\
\hline (French et al., 2008) [25] & $\checkmark$ & & & Institution & YSR/days of marijuana use \\
\hline (Olsson, 2010) [26] & $\checkmark$ & & $\checkmark$ & Societal & - \\
\hline (Sheidow et al., 2012) [27] & $\checkmark$ & & & Institution & TLFB/SRD \\
\hline (Cary et al., 2013) [28] & $\checkmark$ & & & Institution & - \\
\hline Dopp et al. (2014) [30] & $\checkmark$ & & $\checkmark$ & Societal & - \\
\hline Borduin et al. (2015) [31] & $\checkmark$ & & $\checkmark$ & Societal & - \\
\hline
\end{tabular}

$C B C L$ child behavior checklist, GSI global severity index, SRD self-report delinquency scale, TLFB timeline follow-back form, YSR youth self report

should be interpreted with caution. Furthermore, the study of Borduin et al. [31] was based on a very small (the smallest one in this review) sample size (only 48 patients) so uncertainty around the estimates (not reported) is expected to be high. Sensitivity analysis is not a solution for this problem as significance of the results cannot be determined (as the estimates in the sensitivity analysis are also subjected to uncertainty). The juvenile drug court programs, analyzed in the study of Sheidow et al. [27] are not easily generalized to other settings as they show great variation due to absence of a strict format. In addition, other settings may have different populations and salaries implying differences in costs. Almost all studies were cautious with drawing conclusions on their data. They not only recognized limitations within their research but also recognized that the number of economic evaluations is very limited and more research is needed before being able to draw conclusions [22-28, 32].

\section{Meta analysis}

The data from the economic evaluations were not pooled as the population, setting, outcomes, costs and interventions were not comparable across studies.

\section{Discussion}

This systematic review summarized and evaluated the cost-effectiveness of family/family-based therapy for adolescents with externalizing disorders, substance use disorder and delinquency. The overall quality of these studies was low, they produced mixed results.. Research should consider a wider perspective and take into account all relevant costs and effects using sophisticated models. Studies evaluating family/familybased therapy concerned various outcomes and costs, and investigated a variety of treatments in various populations in different settings. Therefore it was not possible to conduct a meta-analysis.

As expected, most of the studies were conducted in the United States where family/family-based treatments originate from $[10,11,34]$. The findings cannot be easily generalized to other health care systems as they differ between countries. The quality assessments showed that overall studies scored between 50 and $86 \%$ and only two studies scored higher than $80 \%[26,28,30,31]$. Studies that were conducted more recently, were in general higher of quality. When the two most recent studies $[30,31]$ were not considered, the quality of the studies overall was slightly higher for those studies originating from Europe. The quality of the two most recent studies was high when using the quality checklists, however, they also contained some important limitations. Firstly, although quality checklists only contain one question with respect to uncertainty around the estimates, it can be of paramount importance, especially when the sample size is low. Secondly, these studies are not easily generalized to an European setting as they conducted costbenefit analyses, opposed to cost-effectiveness analyses that are commonly applied in European studies. Although the checklists used to assess quality of the studies depend on the subjective evaluation of the researchers and have yet not been validated, these two checklists have received much scrutiny and are therefore recommended [18]. Recommendations that follow from the quality assessment of the studies that were included in the review, are the following. Different treatments that are included in the study should be described more 
clearly so the differences and similarities between treatments are understandable. In many of the studies included in the review, the perspective taken was not mentioned or did not match with the categories of the costs that were included. In line with guidelines for economic evaluations the perspective should be stated [33]. A more broad perspective (societal versus healthcare) is recommended. The unit costs and resource use should be reported separately and a source of the references for the unit costs should be given. It is also important to explicitly mention whether a study is considered a cost-effectiveness/cost-benefit or costutility analysis.

Most studies included in the review used no model or simple models (regression). More complex models, like multilevel analysis, should be used. In this way covariates can be included, correlation between measurements over time can be addressed, missing data is accounted for and skewness in the costs and effects is considered. Uncertainty around costs should also be presented by using for instance bootstrapped costs/effects confidence intervals and can be visualized in a cost-effectiveness plane. Sensitivity analysis should be applied to variables that are uncertain (the rationale behind it should be explained). A one way sensitivity analysis is not always sufficient and a sensitivity anaysis also taking into account interactions between variables should be considered. A common discount rate should be applied for all costs and effects. Summary measures of the cost-benefit, cost-effectiveness or cost utility should be given. In case of a cost-effectiveness analysis incremental costeffectiveness ratio (ICERS) should be calculated. For conducting economic evaluations it is advised to consult a health economist.

\section{Conclusions}

Although family/family-based treatments are widely used and can be considered as effective for the treatment of a wide range of disorders [17], costeffectiveness also needs to be addressed. Taking costeffectiveness into account may have a large impact as family/family-based treatments are expensive. This review has summarized the economic evidence of family/family-based therapy for substance use disordersand delinquency in adolescents in a systematic and transparent way by using state of the art guidelines $[18,19]$. As there are few studies evaluating the cost-effectiveness of family/family-based therapy and the quality of the existing studies is limited, new studies using higher quality standards are necessary. Large-scale implementation of these treatment models should be held back, until more evidence is available.

\section{Appendix 1}

Table 7 Description family/family-based interventions

\begin{tabular}{cl}
\hline Family/family-based interventions \\
\hline MST Target family interaction and the extended social systems in \\
youths with substance abuse problems, delinquency or \\
antisocial behavior / Permits separate meetings adolescent \\
but preference for family /More focus on antisocial behavior/ \\
focused both on family functioning and on extra familial \\
functioning / Treatment team not actively involved as \\
observers and actors but team is only self-reflexive/ Treatment \\
team actively involved as observers and actors /degree of \\
severity higher and combination of more problems \\
Target family interaction and the extended social systems in \\
youths with substance abuse problems, delinquency or \\
antisocial behavior/ Almost no separate meetings adolescent \\
/More focus on antisocial behavior/More focused on family \\
functioning less on extra familial functioning/ Treatment team \\
not actively involved as observers and actors but team is only \\
self-reflexive/ explicitly emphasizes therapist is integral part of \\
the system/degree of severity lower \\
Target family interaction and the extended social systems in \\
youths with substance abuse problems, delinquency or \\
antisocial behavior/ Separate meetings adolescent/ Focus on \\
substance abuse / focused both on family functioning and on \\
extra familial functioning /Treatment team not actively \\
involved as observers and actors but team is only self-reflexive/ \\
degree of severity higher
\end{tabular}

Sources: Leukehof et al. and Oudhof et al. (Leukefeld et al., 2008; Oudhof et al., 2009)

MST multisystemic therapy, FFT functional family therapy, MDFT

multidimensional family treatment

\section{Appendix 2}

\section{Search terms Pubmed}

1. "family therapy" $[\mathrm{MESH}]$

2. "Functional family therapy"

3. (FFT NOT ("fast Fourier transform" OR "freedomfrom-transfusion" OR "fast Fourier transforms" OR "fast Fourier transformation" OR "Far-Field Transform"))

4. "Multisystemic Therapy"

5. (MST NOT ("microbial source tracking" OR "minimum spanning tree"))

6. "Multidimensional Treatment Foster Care"

7. "MTFC"

8. "multidimensional family therapy"

9. "MDFT"

10. "family behavior therapy"

11. "FBT"

12. brief strategic family therapy"

13. "BSFT"

14. "family based therapy"[Title/Abstract]

15. "family based interventions"[Title/Abstract]

16. "family based intervention"[Title/Abstract]

17. "family systems intervention" [Title/Abstract]

18. "family systems interventions" [Title/Abstract] 
19. "family system intervention" [Title/Abstract]

20. "family system interventions" [Title/Abstract]

21. "family intervention program"[Title/Abstract]

22. "family intervention programs"[Title/Abstract]

23. "systemic Therapy" [Title/Abstract]

24. OR $1-23$

25. "economic evaluation" [title/Abstract]

26. "economic evaluations" [title/Abstract]

27. "cost effective" [title/Abstract]

28. "cost effectiveness" [title/Abstract]

29. "cost utility analysis" [title/Abstract]

30. "costs" [Title/Abstract] AND "effect"[Title/Abstract]

31. "cost" [Title/Abstract] AND "effect"[Title/Abstract]

32. "cost" [Title/Abstract] AND "effects"[Title/Abstract]

33. "costs" [Title/Abstract] AND "effects"[Title/Abstract]

34. "costs"[Title/Abstract] AND "benefits"[Title/Abstract]

35. "cost" [Title/Abstract] AND "benefit"[Title/Abstract]

36. "costs" [Title/Abstract] AND "benefit"[Title/Abstract]

37. "cost" [Title/Abstract] AND "benefits"[Title/Abstract]

38. "costs" [Title/Abstract] AND "utility"[Title/Abstract])

39. "cost" [Title/Abstract] AND "utility"[Title/Abstract])

40. "cost" [Title/Abstract] AND "utilities"[Title/Abstract]

41."costs" [Title/Abstract] AND "utilities"[Title/Abstract])

42. "Cost Analysis" [title/Abstract]

43. "Cost Measures" [title/Abstract]

44. "cost benefit analysis"[title/Abstract]

45. "cost measure" [title/Abstract]

46. "cost" [title]

47. "costs" [title]

48. "cost benefit analysis" [MESH]

49. OR $25-48$

50. NOT (cancer[Title/Abstract]OR psoriasis[Title/

Abstract]OR "radiation therapy"[Title/Abstract] OR diabetes[Title/Abstract] OR diabetic[Title/Abstract] OR obesity [Title/Abstract] OR aids[Title/Abstract] OR HIV[Title/Abstract] OR sarcomas[Title/Abstract] OR chemotherapy[title/Abstract]))

\section{24 AND 49 AND 50}

\section{Search terms Eric, Psycinfo and Cochrane}

In Eric, the same search terms were used except for the MESH terms. In psycinfo, the MESH terms were replaced with APA's thesaurus of Psychological index Terms and in cochrane, the same terms were used.

\footnotetext{
Abbreviations

ACER, average cost-effectiveness ratio; ACRA, adolescent community reinforcement approach; $A T$, after treatment; $C$, comparator; $C A U$, care as usual; $\mathrm{CBCL}$, child behavior checklist; $\mathrm{CD}$, conduct disorder; CHEC, Consensus on Health Economic Criteria; CM, contingency management; DC, drug court; ENG, England; ERIC, Education Resource information Centre; FC, family court with community services; FFT, functional family therapy; FSN, family support network; Group, skill-focused psycho-education group intervention; GSI, global severity index; I, intervention; ICER, incremental cost-effectiveness ratio; IT, individual treatment; Joint, combination of individual and family therapy; MDFT, multidimensional family therapy; MeSH, Medical Subject Headings; MET/CBT12, motivational enhancement treatment/cognitive behavior
}

therapy, 12 sessions; MET/CBT5, motivational enhancement treatment/cognitive behavior therapy, 5 sessions; MEX, Mexico V; MST, multisystemic therapy; MST-PSB, multisystemic therapy for problem sexual behavior; NA, not applicable; NC, not clear; NHS EED, NHS Economic Evaluation Database; NS, not stated; NS1, reference to non-accessible article; PC, psychiatric crisis; PRISMA, Preferred Reporting for Systematic reviews and Meta-Analyses; RCT, randomized controlled trial; SD, standard deviation; SRD, self-report delinquency scale; SUD, substance use disorder; SW, Sweden; TLFB, timeline follow-back form; UK, United Kingdom; USA, United States of America; USD, United States Dollar; YSR, youth self report

\section{Funding \\ This study was funded by ZonMw, The Netherlands Organization for Health Research and Development. The funder has had no participation in the design and conduct of the study, collection, analysis and interpretation of the data and writing of the manuscript.}

\section{Availability of data and materials}

All data supporting our findings is contained within the manuscript.

\section{Authors' contributions}

$M G$, SJS and CAMB were responsible for screening articles and data extraction. MG designed and completed this systematic review as part of her $\mathrm{PhD}$ project under supervision of LHVR. EvS and $\mathrm{VMH}$ provided input on the literature that was included and the process of systematic reviewing.

All authors contributed to and approved the final manuscript.

\section{Competing interests}

The authors declare that they have no competing interests.

\section{Consent for publication \\ Not applicable.}

Ethics approval and consent to participate

Not applicable.

\section{Author details}

${ }^{1}$ Institute for Medical Technology Assessment \& Institute of Health Policy \& Management, Erasmus University Rotterdam, P.O. Box 17383000 DR Rotterdam, The Netherlands. 'Brijder Addiction Treatment, Parnassia Group, Parnassia Addiction Research Centre (PARC), P.O. Box 530022505 AA The Hague, The Netherlands. ${ }^{3}$ Department of Child and Adolescent Psychiatry, Curium, Leiden University Medical Center, Leiden University, P.O. Box 372300 AA Leiden, The Netherlands.

Received: 3 December 2015 Accepted: 23 June 2016

Published online: 13 July 2016

\section{References}

1. von Sydow K, Retzlaff R, Beher S, Haun MW, Schweitzer J. The efficacy of systemic therapy for childhood and adolescent externalizing disorders: a systematic review of 47 RCT. Fam Process. 2013;52(4):576-618.

2. Baldwin SA, Christian S, Berkeljon A, Shadish WR. The effects of family therapies for adolescent delinquency and substance abuse: a meta-analysis. J Marital Fam Ther. 2012;38(1):281-304.

3. Broman $C L$, Reckase MD, Freedman-Doan CR. The role of parenting in drug use among black, Latino and white adolescents. J Ethn Subst Abuse. 2006; 5(1):39-50.

4. Choquet M, Hassler C, Morin D, Falissard B, Chau N. Perceived parenting styles and tobacco, alcohol and cannabis use among French adolescents: gender and family structure differentials. Alcohol Alcohol. 2008;43(1):73-80.

5. Hoeve M, Dubas JS, Gerris JR, van der Laan PH, Smeenk W. Maternal and paternal parenting styles: unique and combined links to adolescent and early adult delinquency. J Adolesc. 2011;34(5):813-27.

6. Kristjansson AL, Sigfusdottir ID, Allegrante JP. Adolescent substance use and peer use: a multilevel analysis of cross-sectional population data. Subst Abuse Treat Prev Policy. 2013;8:27.

7. Curcio AL, Mak AS, George AM. Do adolescent delinquency and problem drinking share psychosocial risk factors? A literature review. Addict Behav. 2013;38(4):2003-13. 
8. Liddle HA, Rowe CL, Dakof GA, Ungaro RA, Henderson CE. Early intervention for adolescent substance abuse: pretreatment to posttreatment outcomes of a randomized clinical trial comparing multidimensional family therapy and peer group treatment. J Psychoactive Drugs. 2004;36(1):49-63.

9. Carr A. The effectiveness of family therapy and systemic interventions for child-focused problems. J Fam Ther. 2008;31(1):3-45.

10. Henggeler SW, Borduin CM. Family therapy and beyond: A multisystemic approach to treating the behaviour problems of children and adolescents. Pacific Grove: Brooks/Cole; 1990.

11. Alexander JF, Parsons BV. Functional family therapy. Monthery: Brooks/Cole; 1982

12. Liddle HA. Multidimensional Family Therapy for Adolescent Cannabis users In: Cannabis Youth Treatment Series Volume 5. U.S. Department of Health and Human Services. 2002. http://adaiclearinghouse.org/downloads/ Multidimensional-Family-Therapy-for-Adolescent-Cannabis-Users-207.pdf. Accessed 1 July 2013.

13. Retzlaff R, von Sydow K, Beher S, Haun MW, Schweitzer J. The efficacy of systemic therapy for internalizing and other disorders of childhood and adolescence: a systematic review of 38 randomized trials. Fam Process. 2013;52(4):619-52.

14. Hendriks VM, Schee E, Blanken P. Treatment of adolescents with a cannabis use disorder: Main findings of a randomized controlled trial comparing multidimensional family therapy and cognitive behavioral therapy in The Netherlands. Drug Alcohol Depend. 2011;119(1-2):64-71.

15. Van der Stouwe T, Asscher JJ, Stams GJ, Deković M, van der Laan PH. The effectiveness of Multisystemic Therapy (MST): a meta-analysis. Clin Psychol Rev. 2014;34(6):468-81.

16. Darnell AJ, Schuler MS. Quasi-experimental study of functional family therapy effectiveness for juvenile justice aftercare in a racially and ethnically diverse community sample. Child Youth Serv Rev. 2015;50:75-82.

17. Morgan TB, Crane DR. Cost-effectiveness of family-based substance abuse treatment. J Marital Fam Ther. 2010;36(4):486-98.

18. Shemilt I, Mugford M, Byford S, Drummond MF, Eisenstein E, Knapp M, Mallender J, McDaid D, Vale L, Walker D. Chapter 15: Incorporating economics evidence. In: Higgins JPT, Green S, editors. Cochrane Handbook for Systematic Reviews of Interventions Version 5.0.1 (updated September 2008). London: The Cochrane Collaboration; 2008.

19. Liberati A, Altman DG, Tetzlaff J, Mulrow C, Gotzsche PC, loannidis JP, Clarke M, Devereaux PJ, Kleijnen J, Moher D. The PRISMA statement for reporting systematic reviews and meta-analyses of studies that evaluate healthcare interventions: explanation and elaboration. BMJ. 2009;339:b2700.

20. Drummond MF, Jefferson TO. Guidelines for authors and peer reviewers of economic submissions to the BMJ. The BMJ Economic Evaluation Working Party. BMJ. 1996;313(7052):275-83.

21. Evers $\mathrm{S}, \mathrm{Goossens} M$, de Vet $\mathrm{H}$, van Tulder M, Ament A. Criteria list for assessment of methodological quality of economic evaluations: Consensus on Health Economic Criteria. Int J Technol Assess Health Care. 2005;21(2):240-5.

22. French MT, Roebuck MC, Dennis ML, Godley SH, Liddle HA, Tims FM. Outpatient marijuana treatment for adolescents. Economic evaluation of a multisite field experiment. Eval Rev. 2003;27(4):421-59.

23. Sheidow AJ, Bradford WD, Henggeler SW, Rowland MD, Halliday-Boykins C, Schoenwald SK, Ward DM. Treament costs for youths receiving multisystemic therapy or hospitalization after a psychiatric crisis. Psych Serv. 2004;55(5):548-54.

24. McCollister KE, French MT, Sheidow AJ, Henggeler SW, Halliday-Boykins CA. Estimating the differential costs of criminal activity for juvenile drug court participants: challenges and recommendations. J Behav Health Serv Res. 2009;36(1):111-26.

25. French MT, Zavala SK, McCollister KE, Waldron HB, Turner CW, Ozechowski TJ. Cost-effectiveness analysis of four interventions for adolescents with a substance use disorder. J Subst Abuse Treat. 2008;34(3):272-81.

26. Olsson TM. MST with conduct disordered youth in Sweden: Costs and benefits after 2 years. Res Social Work Prac. 2010;20(2):561-71.

27. Sheidow AJ, Jayawardhana J, Bradford WD, Henggeler SW, Shapiro SB. Money Matters: Cost Effectiveness of Juvenile Drug Court with and without Evidence-Based Treatments. J Child Adolesc Subst Abuse. 2012;21(1):69-90.

28. Cary M, Butler S, Baruch G, Hickey N, Byford S. Economic evaluation of multisystemic therapy for young people at risk for continuing criminal activity in the UK. PLoS One. 2013;8(4):e61070.
29. Dennis M, Godley SH, Diamond G, Tims FM, Babor T, Donaldson J, Liddle H, Titus JC, Kaminer Y, Webb C, Hamilton N, Funk R.

The Cannabis Youth Treatment (CYT) Study: Main findings from two randomized trials. J Subst Abuse. 2004;27:197-213.

30. Dopp AR, Borduin CM, Wagner DV, Sawyer AM. The economic impact of multisystemic therapy through midlife: a cost-benefit analysis with serious juvenile offenders and their siblings. J Consult Clin Psychol. 2014;4:694-705.

31. Borduin CM, Dopp AR. Economic Impact of Multisystemic Therapy with Juvenile Sexual Offenders. J Fam Psychol. 2015;29(5):687-96.

32. Schoenwald SK, Ward DM, Henggeler SW, Pickrel SG, Patel H. Multisystemic therapy treatment of substance abusing or dependent adolescent offenders: Costs of reducing incarceration, inpatient and residential treatment. J Child Fam Stud. 1996;5(4):431-44

33. Drummond MF, Sculpher MJ, Torrance GW, O'Brien BJ, Stoddart GL. Methods for the economic evaluation of health care programs. 3rd ed. Oxford: University Press; 2005.

34. Liddle HA. A multidimensional model for treating the adolescent who is abusing alcohol and other drugs. In: Snyder W, Ooms T, editors. Empowering families, helping adolescents: family-centered treatment of adolescents with alcohol, drug abuse and other mental health problems. 1st ed. Washington, DC: United States Public Health Service; 1991.

\section{Submit your next manuscript to BioMed Central and we will help you at every step:}

- We accept pre-submission inquiries

- Our selector tool helps you to find the most relevant journal

- We provide round the clock customer support

- Convenient online submission

- Thorough peer review

- Inclusion in PubMed and all major indexing services

- Maximum visibility for your research

Submit your manuscript at www.biomedcentral.com/submit
C Biomed Central 